University of Konstanz Department of Economics

\title{
Optimal Sales Force Compensation
}

Matthias Kräkel and Anja Schöttner

Working Paper Series 2014-09

http://www.wiwi.uni-konstanz.de/econdoc/working-paper-series/ 


\title{
Optimal Sales Force Compensation
}

\author{
Matthias Kräkel* Anja Schöttner ${ }^{\dagger}$
}

\begin{abstract}
We analyze a dynamic moral-hazard model to derive optimal sales force compensation plans without imposing any ad hoc restrictions on the class of feasible incentive contracts. We explain when the compensation plans that are most common in practice - fixed salaries, quota-based bonuses, commissions, or a combination thereof - are optimal. Fixed salaries are optimal for small revenue-cost ratios. Quota-based bonuses (commissions) should be used if the revenue-cost ratio takes intermediate (large) values. If firms face demand uncertainty, markets are rather thin, and the revenue-cost ratio large, firms should combine a commission with a quota-based bonus. If word-of-mouth advertising affects sales, a dynamic commission that increases over time can be optimal. When entering a new market or launching a new product, firms should install long-term bonus plans.
\end{abstract}

Key Words: sales force compensation, commissions, quota-based bonuses

${ }^{*}$ University of Bonn, Institute for Applied Microeconomics, Business Administration, Adenauerallee 24-42, D-53113 Bonn, Germany, tel: +49 228 733914, fax: +49 228 739210, e-mail: m.kraekel@uni-bonn.de.

${ }^{\dagger}$ University of Konstanz, Department of Economics, Chair of Organizational Economics, Postbox 144, D-78457 Konstanz, Germany, tel: +49 7531 882992, fax: +49 7531 884456, e-mail: anja.schoettner@uni-konstanz.de. 


\section{Introduction}

Personal selling via sales forces is one of the most important marketing instruments. According to Zoltners et al. (2008, p. 115), U.S. firms spend approximately $\$ 800$ billion on sales forces each year - almost three times as much as they spent on advertising in 2006. Sales force compensation plans, however, differ across firms. Joseph and Kalwani (1998, p. 149) report that $5 \%$ of the 266 companies participating in a survey exclusively pay fixed salaries to their salespeople, $24 \%$ use only commissions, $37 \%$ use only a bonus component, and $35 \%$ use both commissions and bonus pay. By far the most important criterion in determining bonus payments was the comparison of actual sales and a predetermined quota. Hence, commissions and/or quota-based bonuses appear to be the most common forms of sales force compensation.

In this paper, we analyze an agency-theoretic framework that can explain under which conditions one form of compensation plan dominates the other. We employ a dynamic moralhazard model that allows us to solve for the optimal compensation plan without imposing any ad hoc restrictions on the class of feasible contracts. We find that those compensation schemes that are frequently observed in practice - fixed salary, quota bonus, commission, or a combination thereof - indeed often maximize firm profits. In particular, we are the first to show that combining a commission with a quota-based bonus can be an optimal compensation scheme.

In our model, a sales agent has to decide whether to work hard or not to sell a product in each of two periods. Both the agent and the firm observe sales at the end of each period. The agent is protected by limited liability so that his compensation must be non-negative. ${ }^{1}$ For our basic model, we obtain the following results. If the ratio between sales revenues and the agent's effort costs is sufficiently low, the firm pays only a fixed salary that induces low effort in both periods. For intermediate revenue-cost ratios, the firm wants to avoid that the agent always exerts low effort. Optimal compensation is then given by the class of cutoff incentive plans that make the agent work hard in the first period, but lead to high effort in the second period if and only if a first-period sale occurred. A central feature of such an incentive plan is that the agent must sell the product in the first period to earn a reward. A quota-based bonus scheme which offers a bonus to the agent if and only if he was successful in both periods belongs to this class of contracts.

For high revenue-cost ratios, optimal compensation is described by the class of permanent incentive plans that always induce high effort. The well-known commission scheme, which

\footnotetext{
${ }^{1}$ This assumption is common in the literature. See, e.g., Sappington (1983), Innes (1990), Demougin and Fluet (1998), Oyer (2000), Simester and Zhang (2010), Simester and Zhang (2013), Poblete and Spulber (2012), and Kishore et al. (2013).
} 
pays a certain commission rate per sale to the agent, belongs to this class of incentives plans. Importantly, the quota-based bonus and the commission are mutually exclusive as optimal sales force compensation. The advantage of the quota-based bonus over the commission is that the former induces high effort in the first period at lower cost for the firm. However, this also implies that the agent might be demotivated in the second period if it is clear that he cannot make the quota. The firm accepts this drawback only if the potential loss, indicated by the revenue-cost ratio, is not too large. Moreover, for a given revenue-cost ratio, a quota-based bonus is optimal if sales respond either sufficiently little or sufficiently strongly to the agent's effort.

We also consider two variants of our basic model - demand uncertainty and preference uncertainty. In the former case, future demand cannot be perfectly foreseen and may depend on previous sales. We show that, when the firm wishes to induce high effort in both periods, demand uncertainty can imply the optimality of an incentive compensation plan that combines a commission with a quota-based bonus. This is the case when the product market is rather thin, i.e., a first-period sale makes it harder to sell the product in the second period. By contrast, if a first-period sale makes a second-period sale more likely (e.g., due to word-of-mouth advertising), a dynamic commission that increases over time is optimal. We further find that, under demand uncertainty, a commission scheme can exhibit another comparative advantage because the firm might be able to implement the optimal commission even if it has less knowledge on short-term demand developments than the sales agent. In such a situation, the commission is the uniquely optimal permanent incentive plan. Moreover, we show that the three practically most relevant incentive plans - commission, bonus, and a combination thereof - are all unique solutions to the firm's optimization problem when the firm collects sales figures less frequently and hence observes only total sales at the end of the second period. Interestingly, scarcer information on sales performance has no adverse effect on firm profits because the aforementioned incentive plans do not require intermediate sales information.

Preference uncertainty reflects a situation where it is uncertain how customers respond to the sales agent's effort, e.g., because the firm enters a new market or launches a new product. In this case, a commission is no longer optimal. Instead, the firm maximizes profits by either a fixed salary, a quota bonus, or an incentive scheme that focuses on second-period sales. Finally, we discuss how advertising, the infeasibility of long-term contracts, and limited liability of the firm affect the optimal compensation plan. In the latter two situations, the quota bonus as an optimal sales force compensation turns out to be less robust. Our results can be nicely translated into clear empirical predictions, which we discuss in the conclusion.

Our paper closes a gap in the theoretical literature on optimal sales force compensation by 
providing optimality conditions for the different types of incentive compensation plans that are typically observed in practice. The analysis of optimal sales force compensation under moral hazard traces back to Basu et al. (1985). They show for a single-period setting with a risk-averse agent that optimal incentive pay usually is a non-linear increasing function of sales. It is argued that commonly used compensation plans can be seen as a piecewise linear approximation of their optimal contract. Raju and Srinivasan (1996) show that such an approximation indeed only leads to a small loss relative to the optimal contract.

By now, there is an extensive literature analyzing optimal single-period sales force compensation in various contexts (see Coughlan (1993) and Albers and Mantrala (2008) for surveys). Dearden and Lilien (1990) and Lal and Srinivasan (1993) extend the work by Basu et al. (1985) to dynamic environments. Dearden and Lilien (1990) explain how the compensation plan should be adjusted in the presence of production learning effects. Lal and Srinivasan (1993) consider a setting that satisfies the properties for the optimality of a linear incentive scheme as characterized by Holmstrom and Milgrom (1987). By contrast, we provide conditions under which non-linear incentive schemes (e.g., quota-based bonuses) dominate linear ones (e.g., commissions) in a dynamic setting. ${ }^{2}$ Focussing on a behavioral approach, Jain (2012) shows that multiperiod quotas can solve a self-control problem on the side of the agent. Our results offer an alternative explanation for the optimality of quotas based on the rational behavior of sales agents. Kishore et al. (2013) show that quota-based bonuses exhibit an advantage over commissions when multitasking concerns are present but do not solve for the optimal contract. In a recent empirical study, Chung et al. (2013) find that quota-based bonuses enhance performance in a firm that also uses commissions. Our theoretical model confirms this observation for situations with demand uncertainty.

The binary-effort approach used in this paper is widely accepted in agency theory (e.g., Che and Yoo (2001); Laffont and Martimort (2002); Schmitz (2005, 2013); Simester and Zhang (2010); Kaya and Vereshchagina (2014)). ${ }^{3}$ Our paper is particularly related to dynamic binaryeffort models with moral hazard and limited liability, which are analyzed by Bierbaum (2002) and Schmitz $(2005,2013)$. In contrast to our paper, these works focus on a situation where the principal wishes to induce high effort in each period. Thus, by assumption, a quota cannot be optimal because it would lead to low effort when it turns out that the quota cannot be made. ${ }^{4}$ As another difference to our setting, Bierbaum (2002) assumes that the principal can

\footnotetext{
${ }^{2}$ Oyer (2000) shows that a bonus tied to a quota can be the uniquely optimal contract in a static setting. Also employing a static model, Bak and Klecz-Simon (2013) find that introducing quota bonuses for sales agents engaging in Bertrand competition can increase firm profit. Agents, however, incur a constant cost per sale and do not choose effort.

${ }^{3}$ Continuous effort would not be analytically tractable in our dynamic setting.

${ }^{4}$ Our result that the principal may wish to induce high effort only after a first-period success resembles a
} 
extract payments from the agent up to his first-period wage in the second period, or dismiss the agent after the first period. In Schmitz $(2005,2013)$, the first-period outcome affects the effectiveness of effort in the second stage. By contrast, there are no externalities across periods in our model. The only exception is that, under demand uncertainty, the probability that there will be a customer in the second period can depend on first-period sales.

The remainder of the paper is organized as follows. Section 2 introduces the basic model. In Section 3, we solve this model and show that fixed salaries, quotas, and commissions are optimal contracts that are mutually exclusive. Sections 4 and 5 consider demand uncertainty and preference uncertainty as extensions of the basic model and investigate the robustness of the previously optimal contracts. Section 6 discusses further variations of the basic model. Section 7 concludes.

\section{The Basic Model}

A firm hires a sales agent to sell its service or product in each of two periods. In each period $i$ $(i=1,2)$, the sales agent interacts with one customer. When dealing with customer $i$, the agent can exert low or high effort $e_{i} \in\{0,1\}$. The firm cannot observe the agent's effort choice so that the given setting describes a typical moral-hazard problem. We apply the usual tie-breaking rule that the agent will choose high effort if he is indifferent between both effort levels. The probability that a customer buys the product is $\alpha$ when effort is low and $\alpha+\rho$ when effort is high, with $\alpha, \rho>0$ and $\alpha+\rho<1 .^{5}$ The parameter $\rho$ thus reflects how responsive sales are to the agent's effort. Effort costs are $c e_{i}$, where $c>0$. When a sale occurs, the firm receives the revenue $R>0$. We assume that $\rho R-c>0$, i.e., exerting high effort is efficient.

Let $x_{i} \in\{0,1\}$ indicate whether customer $i$ bought the product $\left(x_{i}=1\right)$ or not $\left(x_{i}=0\right)$. The total number of sales is $x=x_{1}+x_{2}, x \in\{0,1,2\}$. At the end of period $i$, both the firm and the agent observe $x_{i}{ }^{6}$ At the beginning of the first period, the firm offers the agent a compensation scheme $w$ that depends on sales, $w=\left(w_{00}, w_{10}, w_{01}, w_{11}\right)$, where $w_{x_{1} x_{2}}$ denotes the payment when sales $x_{1}, x_{2} \in\{0,1\}$ occurred. The firm designs the compensation scheme to maximize expected profit, i.e., expected revenues minus expected compensation. Note that our setting allows for a variety of compensation plans, including fixed salaries, commissions, and quota-based bonuses.

To exclude trivial solutions to the given moral-hazard problem, we assume that the firm

finding by Ohlendorf and Schmitz (2012), who analyze a dynamic limited-liability model with continuous effort. In their framework, an optimal dynamic contract always exhibits memory.

${ }^{5}$ We exclude $\alpha=0$ because in this case the principal can always induce the efficient (first-best) solution.

${ }^{6}$ In Section 6, we discuss a situation where the firm only observes total sales $x$. 
faces some contractual friction. Contract theory offers two standard frictions (e.g., Laffont and Martimort (2002), Sections 4.3 and 4.4) - the agent is assumed to be either risk averse (and unlimitedly liable) or protected by limited liability (and risk neutral). Both frictions imply that providing incentives leads to costs for the firm which exceed the effort and opportunity costs of the agent. In case of a risk-averse agent, the firm has to compensate him for any income risk he bears. In case of limited liability, the firm has to leave a rent to the agent. In our setting, we assume that the sales agent is risk neutral ${ }^{7}$ but protected by limited liability in terms of $w_{x_{1} x_{2}} \geq 0$ for all $x_{1}$ and $x_{2}$ (i.e., the firm cannot impose negative wages to punish the agent for poor performance). The agent's reservation utility is zero. ${ }^{8}$

The timeline of the game is as follows. First, the firm offers a compensation contract. Then the sales agent accepts or rejects the contract offer. If he rejects, the game will end and the agent earns his reservation value. If he accepts, he will interact with the first-period customer and decide on effort. After having observed the first-period sales outcome, the agent deals with another customer in the second period and again chooses high or low effort. Finally, the sales agent is paid according to the compensation contract.

\section{Optimal Compensation in the Basic Model}

When determining the optimal compensation for the sales agent, the firm may be interested in different effort levels depending on the specific nature of the transaction, which is captured by the values of the model parameters. Therefore, we first consider three scenarios characterized by the implementation of different effort combinations. Each scenario will turn out optimal for certain parameter constellations.

In scenario 1 , the firm induces low effort in both periods $\left(e_{1}=e_{2}=0\right)$. The firm then optimally offers a fixed salary of zero because both the agent's reservation utility and the loweffort costs are normalized to zero. Optimal compensation thus is $w^{F}=(0,0,0,0)$ and the firm's expected profits are $\pi^{F}=2 \alpha R$.

By contrast, in scenario 2, effort is high in both periods $\left(e_{1}=e_{2}=1\right)$. We label the corresponding class of optimal contracts as permanent incentive plans since they provide high effort incentives independent of the sales history. The firm's contracting problem is solved by backward induction. To this end, consider the agent's effort choice with the second customer. To implement $e_{2}=1$ for either first-period outcome, the wages $w_{x_{1} x_{2}}$ have to satisfy the second-

\footnotetext{
${ }^{7}$ The empirical findings of Ackerberg and Botticini (2002), Hilt (2008), and Bellemare and Shearer (2010) show that agents with low risk aversion sort themselves into risky jobs. Hence, it is not unrealistic to assume that sales agents, in particular, have a high risk tolerance in practice.

${ }^{8}$ This assumption will ensure that the firm has to leave a rent to the agent under any incentive contract.
} 
period incentive constraint

$$
(\alpha+\rho) w_{x_{1} 1}+(1-\alpha-\rho) w_{x_{1} 0}-c \geq \alpha w_{x_{1} 1}+(1-\alpha) w_{x_{1} 0} \text { for } x_{1}=0,1,
$$

which requires that the agent's expected net income is greater or equal when choosing high instead of low effort, irrespective of the sales history $x_{1}$. The incentive constraint is equivalent to

$$
w_{x_{1} 1} \geq \frac{c}{\rho}+w_{x_{1} 0} \text { for } x_{1}=0,1 .
$$

Anticipating high effort with the second customer, the agent also chooses high effort with the first customer (i.e., $e_{1}=1$ ) when this leads to a higher expected net income across both periods than low first-period effort. The corresponding first-period incentive constraint is

$$
\begin{gathered}
(\alpha+\rho)\left[(\alpha+\rho) w_{11}+(1-\alpha-\rho) w_{10}-c\right]+(1-\alpha-\rho)\left[(\alpha+\rho) w_{01}+(1-\alpha-\rho) w_{00}-c\right]-c \\
\geq \alpha\left[(\alpha+\rho) w_{11}+(1-\alpha-\rho) w_{10}-c\right]+(1-\alpha)\left[(\alpha+\rho) w_{01}+(1-\alpha-\rho) w_{00}-c\right] \\
\Leftrightarrow \rho\left[(\alpha+\rho)\left(w_{11}-w_{01}\right)+(1-\alpha-\rho)\left(w_{10}-w_{00}\right)\right] \geq c .
\end{gathered}
$$

The firm wants to induce permanent incentives at minimal costs. Hence it solves

$$
\min _{w_{11}, w_{10}, w_{01} w_{00} \geq 0}(\alpha+\rho)^{2} w_{11}+(\alpha+\rho)(1-\alpha-\rho) w_{10}+(1-\alpha-\rho)(\alpha+\rho) w_{01}+(1-\alpha-\rho)^{2} w_{00}
$$

subject to the incentive constraints (2) and (3) and the participation constraint that guarantees the agent a non-negative expected net income. Since non-negative wages are ensured by the limited-liability constraint $w_{11}, w_{10}, w_{01}, w_{00} \geq 0$, the agent can always achieve a non-negative net income by accepting the contract and choosing zero effort. Thus, we can ignore the participation constraint in the following.

The optimal solution to the firm's problem comprises $w_{00}=0$, which implies $w_{01}=\frac{c}{\rho}$. From the first-period incentive constraint (3), it follows that the firm cannot do better than setting $w_{11}$ and $w_{10}$ such that

$$
(\alpha+\rho) w_{11}+(1-\alpha-\rho) w_{10}=\frac{c}{\rho}+(\alpha+\rho) w_{01}=(1+\alpha+\rho) \frac{c}{\rho} .
$$

The class of optimal permanent incentive plans is thus described by $w_{00}=0, w_{01}=\frac{c}{\rho}$, and any combination of $w_{11}$ and $w_{10}$ satisfying (5) and (2). One possibility is to set $w_{10}=\frac{c}{\rho}$ and $w_{11}=2 \frac{c}{\rho}$, which describes a commission scheme where the sales agent receives the commission $\frac{c}{\rho}$ per sale. The compensation scheme $w^{C}=\left(0, \frac{c}{\rho}, \frac{c}{\rho}, 2 \frac{c}{\rho}\right)$ is thus optimal and the firm's expected 
profit is

$$
\pi^{C}=2(\alpha+\rho) R-(\alpha+\rho)\left[(1+\alpha+\rho) \frac{c}{\rho}+(1-\alpha-\rho) \frac{c}{\rho}\right]=2(\alpha+\rho)\left(R-\frac{c}{\rho}\right) .
$$

Because the agent is protected by limited liability, he earns a rent in this scenario, i.e., the agent's expected wage minus effort costs is strictly larger than his reservation utility. The rent amounts to $2\left[(\alpha+\rho) \frac{c}{\rho}-c\right]=2 \alpha \frac{c}{\rho}$.

In scenario 3, the agent's second-period incentives depend on the sales outcome of the first period. The firm induces $e_{1}=1$ but implements $e_{2}=1$ if and only if $x_{1}=1$. That is, the agent should exert high effort with the second customer if and only if he could sell the product to the first customer. We term the corresponding class of optimal contracts cutoff incentive plans since, as we will show below, the agent must be successful with the first customer as a necessary condition to earn a reward.

From condition (2), we can deduce the second-period incentive constraints

$$
w_{11} \geq \frac{c}{\rho}+w_{10} \quad \text { and } \quad w_{01}<\frac{c}{\rho}+w_{00}
$$

The first-stage incentive constraint differs from constraint (3) above, because the agent anticipates that second-period effort will not always be high:

$$
\begin{gathered}
(\alpha+\rho)\left[(\alpha+\rho) w_{11}+(1-\alpha-\rho) w_{10}-c\right]+(1-\alpha-\rho)\left[\alpha w_{01}+(1-\alpha) w_{00}\right]-c \\
\geq \alpha\left[(\alpha+\rho) w_{11}+(1-\alpha-\rho) w_{10}-c\right]+(1-\alpha)\left[\alpha w_{01}+(1-\alpha) w_{00}\right] \\
\Leftrightarrow \rho\left[(\alpha+\rho) w_{11}+(1-\alpha-\rho) w_{10}-\alpha w_{01}-(1-\alpha) w_{00}\right] \geq(1+\rho) c .
\end{gathered}
$$

Again, the firm minimizes wage costs for implementing the given effort combination. It now solves

$$
\min _{w_{11}, w_{10}, w_{01}, w_{00} \geq 0}(\alpha+\rho)^{2} w_{11}+(\alpha+\rho)(1-\alpha-\rho) w_{10}+(1-\alpha-\rho) \alpha w_{01}+(1-\alpha-\rho)(1-\alpha) w_{00}
$$

subject to (7) and (8). As an immediate consequence, we obtain $w_{00}=w_{01}=0$. A commission scheme can therefore not be optimal. By the incentive constraints (7) and (8), all $w_{11}$ and $w_{10}$ satisfying

$$
(\alpha+\rho) w_{11}+(1-\alpha-\rho) w_{10}=(1+\rho) \frac{c}{\rho} \quad \text { and } \quad w_{11} \geq \frac{c}{\rho}+w_{10}
$$

minimize the firm's wage costs. Therefore, $w_{00}=w_{01}=0$ and the conditions (10) characterize the class of optimal cutoff incentive plans. One optimal plan is $w_{10}=0$ and $w_{11}=\frac{1+\rho}{\alpha+\rho} \frac{c}{\rho}$, which 
corresponds to a quota-based bonus where the agent only earns a bonus if he sold the product to both customers. Hence, the compensation scheme $w^{Q}=\left(0,0,0, \frac{1+\rho}{\alpha+\rho} \frac{c}{\rho}\right)$ is optimal and the firm obtains the expected profit

$$
\begin{aligned}
\pi^{Q} & =(\alpha+\rho) R+\left[(\alpha+\rho)^{2}+(1-\alpha-\rho) \alpha\right] R-(\alpha+\rho)(1+\rho) \frac{c}{\rho} \\
& =(\alpha+\rho)\left(R-\frac{c}{\rho}\right)+\left[(\alpha+\rho)^{2}+(1-\alpha-\rho) \alpha\right] R-(\alpha+\rho) c .
\end{aligned}
$$

The agent earns the rent $\alpha \frac{c}{\rho}$.

Besides the three scenarios we have discussed so far, there are other effort combinations that can be implemented. From the firm's point of view, however, none of the other combinations is optimal. ${ }^{9}$ We thus have three candidate solutions that may lead to maximum firm profits - a fixed-salary in scenario 1, a commission in scenario 2, and a quota-based bonus in scenario 3 . In scenarios 2 and 3, the optimal incentive compensation plan is not unique. However, commission and quota-based bonus are mutually exclusive: In scenario 2 (scenario 3), the uniquely optimal wage if only the second customer buys the product is $w_{01}=\frac{c}{\rho}\left(w_{01}=0\right)$. Thus, the firm will not use a quota scheme in scenario 2 and it will not employ a commission in scenario 3. Comparing firm profits in the three scenarios and defining the thresholds

$$
\Omega_{L}(\alpha, \rho):=\frac{\alpha+\rho}{1+\alpha+\rho} \frac{1+\rho}{\rho^{2}}<\frac{\alpha+\rho}{1-(\alpha+\rho)} \frac{1-\rho}{\rho^{2}}=: \Omega_{H}(\alpha, \rho)
$$

leads to the following result.

Proposition 1 The firm maximizes expected profits by paying a fixed salary, or using a quotabased bonus, or paying a commission. The fixed salary $w^{F}=(0,0,0,0)$ is optimal if and only if $\frac{R}{c} \leq \Omega_{L}(\alpha, \rho)$; the quota-based bonus $w^{Q}=\left(0,0,0, \frac{1+\rho}{\alpha+\rho} \frac{c}{\rho}\right)$ is optimal if and only if $\Omega_{L}(\alpha, \rho)<\frac{R}{c}<\Omega_{H}(\alpha, \rho)$; and the commission $w^{C}=\left(0, \frac{c}{\rho}, \frac{c}{\rho}, 2 \frac{c}{\rho}\right)$ is optimal if and only if $\Omega_{H}(\alpha, \rho) \leq \frac{R}{c}$. The agent earns the rent $\alpha \frac{c}{\rho}$ under the quota-based bonus and the rent $2 \alpha \frac{c}{\rho}$ under the commission.

\section{Proof. See Appendix.}

Proposition 1 shows that one of three practically relevant compensation schemes maximizes the firm's expected profit. Figure 1 illustrates the firm's optimal choice as a function of $R / c$ and $\rho .{ }^{10}$ Eliciting high effort from the agent in at least one period is only worthwhile if the revenue-cost ratio, $R / c$, is sufficiently large, $R / c>\Omega_{L}(\alpha, \rho)$. This condition is also satisfied

\footnotetext{
${ }^{9}$ See the Additional Material 1.

${ }^{10}$ Recall that, by assumption, high effort is efficient, i.e., $\rho R-c>0$. Because this implies $R / c>1 / \rho$, only parameter constellations above the hyperbola $1 / \rho$ are feasible.
} 


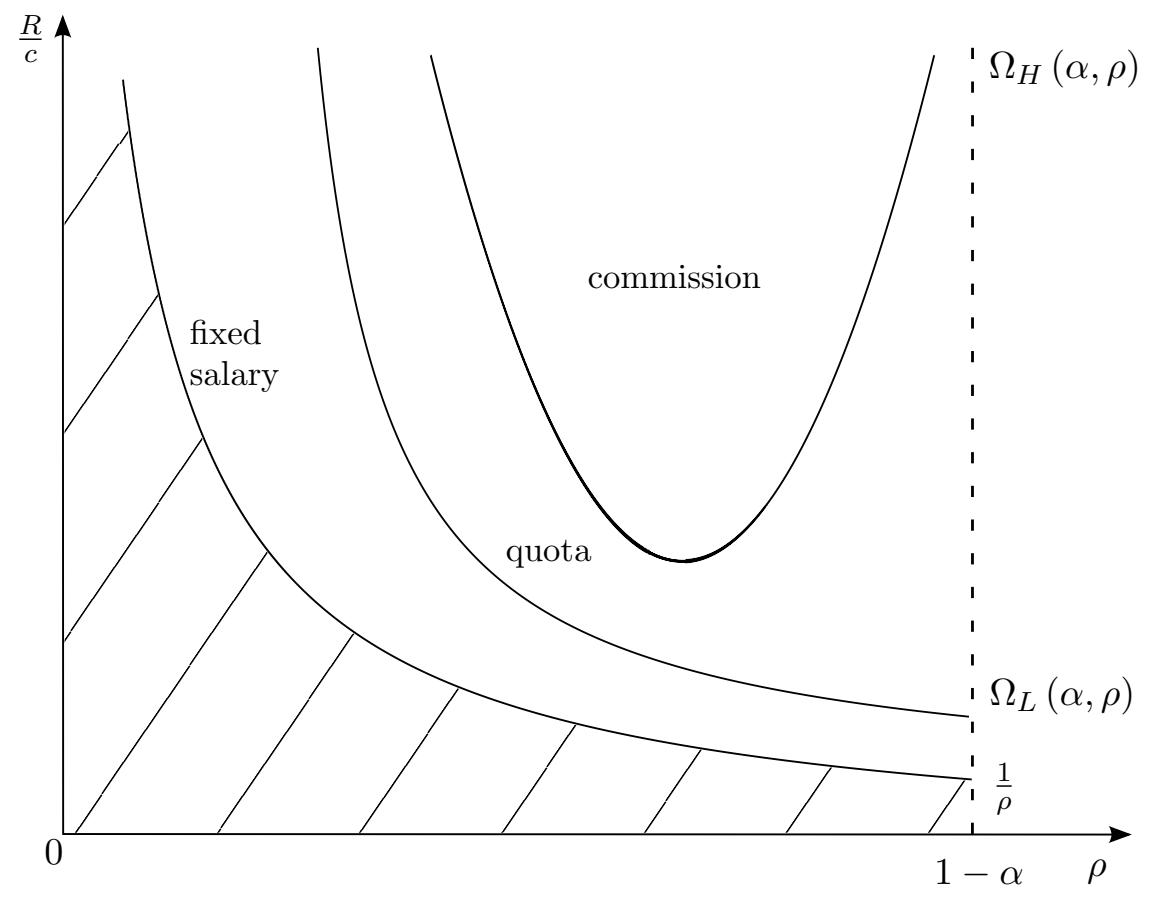

Figure 1: Optimal compensation plans

when $\alpha$ is sufficiently low. Intuitively, the firm wants to provide incentives when a customer is unlikely to buy if the agent does not put forth an extra effort. Furthermore, the firm will implement incentive pay if $\rho$ is sufficiently large, i.e., if sales are highly responsive to effort. The sales agent then responds more strongly to incentives, which makes inducing high effort less costly for the firm because the rent it has to leave to the agent decreases under either incentive scheme.

To provide incentives, the firm can choose between a commission and a quota-based bonus. It uses a commission if it wants to generate strong incentives, i.e., if effort should be high with every customer. In contrast, the quota-based bonus creates only intermediate incentives. ${ }^{11}$ Because the agent is rewarded only when he sold the product to both customers, he will no longer be motivated to exert high effort when he failed to make a sale in the first period. Our results show that such a demotivation effect is frequently in the firm's interest. ${ }^{12}$

The comparative advantage of the quota-based bonus is that the relatively high probability of no reward payment makes incentive provision less costly for the firm: the agent's rent under the quota-based bonus is only half as large as his rent under the commission. Still, the agent exerts high effort with probability $\alpha+\rho$ in the second period under the quota-based bonus. On

\footnotetext{
${ }^{11}$ Kishore et al. (2013) analyze data from a pharmaceutical corporation that switched from a bonus plan to a commission plan to compensate its sales force. They find that the commission plan led to more effective incentives. Overall productivity increased by $24 \%$.

${ }^{12}$ Steenburgh (2008) empirically analyzes the effects of bonuses on sales force performance. He concludes that "[...] bonuses cause some salespeople, those who are unlikely to make quota, to reduce effort, but this effect is more than compensated for by productive increases in output by other salespeople." (Steenburgh 2008, p. 252)
} 
the downside, however, expected revenues are lower under the bonus than under the commission because the chance of a sale in the second period is lower under the bonus. Therefore, a bonus is optimal when $R / c$ is sufficiently small, implying that the loss in revenues is dominated by the decrease in compensation. This is the case if and only if $\frac{R}{c}<\Omega_{H}(\alpha, \rho)=\frac{\alpha+\rho}{1-(\alpha+\rho)} \frac{1-\rho}{\rho^{2}}$. The

higher $\alpha+\rho$ and thus $\frac{\alpha+\rho}{1-(\alpha+\rho)}$, the more likely the first customer is to buy and, consequently, the quota-based bonus does not lead to a loss in revenues because effort will also be high in the second period. A higher $\alpha$ thus makes a bonus more attractive compared to a commission. For $\rho$, however, there is a counteracting effect, reflected by $\frac{1-\rho}{\rho^{2}}$. The larger $\rho$, the easier the agent is to motivate. Therefore, always inducing high effort by a commission becomes more attractive. However, if $\rho$ is sufficiently large, high effort is almost certain to result in a sale. This makes a loss with the second customer highly unlikely under a quota scheme and, thus, the bonus dominates the commission: $\lim _{\rho \rightarrow 1-\alpha} \Omega_{H}(\alpha, \rho)=\infty$. Consequently, firms with sufficiently low or sufficiently high effort-sales responsiveness should adopt a quota-based bonus. This nonmonotonicity result concerning the impact of $\rho$ on the optimal incentive scheme is illustrated in Figure 1 and summarized in the following corollary.

Corollary 1 Assume that the firm wants to provide effort incentives, i.e., $\frac{R}{c}>\Omega_{L}(\alpha, \rho)$. A quota-based bonus is optimal if sales respond either sufficiently little or sufficiently strongly to effort.

The following two sections consider more complex scenarios to check the robustness of our previous findings.

\section{Demand Uncertainty}

In the basic model, we have assumed that the agent's opportunities to sell the product are constant over time, i.e., the agent can be certain that there will be a customer also in the second period. However, in practice firms and their sales agents often face demand uncertainty, i.e., they cannot perfectly foresee future market developments for their product. Moreover, from working in the field the agent is often better informed about short-run demand developments than the firm.

To capture this problem, we now assume that first-period demand is certain but the second customer only arrives with an exogenously given probability $\beta_{x_{1}} \in(0,1)$ where $x_{1} \in\{0,1\}$ again denotes first-period sales. There are three possibilities. We can have $\beta_{1}=\beta_{0}$ so that second-period demand is uncertain but independent of whether the agent was successful with the first customer or not. Alternatively, second-period demand depends on whether there was 
a first-period sale. In that case, we may either have $\beta_{1}>\beta_{0}$ indicating that first-period success increases the probability of a second-period customer compared to a first-period failure, e.g., due to word-of-mouth advertising. Or we may have $\beta_{1}<\beta_{0}$, e.g., because the market has only few potential customers ("thin markets") so that a successful sale in the first period leads to a significant reduction of the remaining market capacity (e.g., markets like real estate markets or the high-end art market where very expensive goods are traded).

The time structure of the model changes as follows: First, the firm decides on the compensation plan. Then, the first customer arrives and the agent decides on exerting effort. Afterwards, the agent (but not the firm) observes whether there will be a second customer (with probability $\beta_{x_{1}}$ ) or not (with probability $1-\beta_{x_{1}}$ ). If a customer arrives, the agent again decides on effort. The firm does not learn whether second-period failure is the result of no demand.

Define

$$
\hat{\Omega}_{L}:=\frac{1+\rho \beta_{1}}{1-\alpha \beta_{0}+(2 \alpha+\rho) \beta_{1}} \frac{\alpha+\rho}{\rho^{2}} .
$$

Solving for the optimal compensation plan leads to the following findings:

Proposition 2 Suppose there is demand uncertainty such that the second-period customer arrives with probability $\beta_{x_{1}} \in(0,1)$. Assume further that $\beta_{0} \leq 2 \beta_{1}$, i.e., the market is not too thin. (i) If $\frac{R}{c} \leq \hat{\Omega}_{L}$, the fixed salary $w^{F}$ is optimal. (ii) If $\hat{\Omega}_{L}<\frac{R}{c} \leq \Omega_{H}(\alpha, \rho)$, the quota-based bonus $w_{d u}^{Q}=\left(0,0,0, \frac{1+\rho \beta_{1}}{(\alpha+\rho) \beta_{1}} \frac{c}{\rho}\right)$ is optimal. (iii) If $\frac{R}{c}>\Omega_{H}(\alpha, \rho)$ and $\beta_{0} \geq \beta_{1}$, the scheme $w_{d u}^{B C}=\left(0, \frac{c}{\rho}, \frac{c}{\rho}, 2 \frac{c}{\rho}+\frac{\alpha}{\alpha+\rho} \frac{\beta_{0}-\beta_{1}}{\beta_{1}} \frac{c}{\rho}\right)$ is optimal. That is, the firm maximizes its profits by using a commission combined with a bonus to be paid when the agent was successful in both periods. If $\frac{R}{c}>\Omega_{H}(\alpha, \rho)$ and $\beta_{0}<\beta_{1}$, the dynamic commission $w_{d u}^{S C}=$ $\left(0,\left[1+\alpha\left(\beta_{0}-\beta_{1}\right)\right] \frac{c}{\rho}, \frac{c}{\rho},\left[2+\alpha\left(\beta_{0}-\beta_{1}\right)\right] \frac{c}{\rho}\right)$ is optimal.

Proof. See Appendix.

Proposition 2 considers a situation where markets are not too thin, i.e., $\beta_{0}$ is not too large relative to $\beta_{1}$. The proof of the proposition shows that we have a similar solution as in the basic model. The firm offers the fixed salary $w^{F}$ if the revenue-cost ratio, $R / c$, is sufficiently small. If the revenue-cost ratio takes intermediate values, optimal sales force compensation is given by a class of cutoff incentive plans that never reward the agent if he failed in the first period (i.e., $w_{01}=0$ ). In particular, the firm cannot do better than using a quota-based bonus that is adapted to the given situation with demand uncertainty. This bonus, $w_{11}$, decreases in $\beta_{1}$ because a low probability $\beta_{1}$ makes it more difficult for the sales agent to obtain the bonus, which requires sales to two customers. This effect demotivates the agent. By choosing a high 
bonus, the firm restores the agent's incentives. ${ }^{13}$

For a sufficiently large revenue-cost ratio, the firm relies again on a class of permanent incentive plans, i.e., it always induces high effort. However, in contrast to the basic model, this class of permanent incentive plans includes a pure commission only if there are no demand externalities across periods (i.e., $\beta_{0}=\beta_{1}$ ). If markets are thin (i.e., $\beta_{0}>\beta_{1}$ ), the firm maximizes profits by combining the commission from the basic model, $\frac{c}{\rho}$, with the bonus $\frac{\alpha}{\alpha+\rho} \frac{\beta_{0}-\beta_{1}}{\beta_{1}} \frac{c}{\rho}$ that is paid when the agent sold the product in both periods. If $\beta_{0}>\beta_{1}$, a first-period failure is quite attractive for the agent since it leads to a higher probability of earning a second-period rent. To work against this detrimental incentive effect, the firm needs to attach an extra reward to two sales in order to incentivize the agent in both periods. By contrast, if $\beta_{0}<\beta_{1}$, the firm can employ a dynamic commission with commission rates that increase over time. ${ }^{14}$ It is no longer optimal for the firm to pay the commission $\frac{c}{\rho}$ for a first-period sale. The agent's payoff from a first-period success can be lowered because the agent is also motivated by the prospect to earn a second-period rent with a higher probability when he is successful in the first period.

The lower threshold for implementing a quota-based bonus, $\hat{\Omega}_{L}$, increases in $\beta_{0}$ and decreases in $\beta_{1}$. Intuitively, inducing high effort in period 1 is less (more) worthwhile, if it lowers (increases) the chance of having a second-period customer relative to low effort. The higher threshold for implementing a bonus, $\Omega_{H}(\alpha, \rho)$, is independent of $\beta_{0}$ and $\beta_{1}$, and identical to the corresponding threshold in the basic model. The proof of Proposition 2 shows that the expected returns and the expected labor costs increase in $\beta_{0}$ and $\beta_{1}$ under both classes of incentive plans and that these effects cancel each other out when the respective profits are compared.

For $\beta:=\beta_{1}=\beta_{0}$, the class of permanent incentive plans from Proposition 2 (iii) contains the commission $w^{C}$ from the basic model, which is independent of $\beta$. The commission scheme thus is a quite robust incentive device when there is demand uncertainty that is independent of the sales history. Moreover, we obtain the following uniqueness result.

Corollary 2 Assume that $\frac{R}{c}>\Omega_{H}(\alpha, \rho)$ and let $\beta:=\beta_{1}=\beta_{0}$. Suppose the firm does not observe $\beta$. The commission $w^{C}$ then is the uniquely optimal sales force compensation because it is the only optimal incentive scheme whose implementation does not require knowledge of $\beta$. If $\hat{\Omega}_{L}<\frac{R}{c} \leq \Omega_{H}(\alpha, \rho)$, the firm always requires knowledge of $\beta$ to determine the optimal compensation.

Proof. See Appendix.

\footnotetext{
${ }^{13}$ Note that the bonus $w_{11}$ approaches infinity as $\beta_{1}$ goes to zero. A very large bonus may, thus, not be feasible if the firm is wealth constrained. We discuss this problem in more detail in Section 6.4.

${ }^{14}$ Note that $w_{11}=w_{10}+w_{01}$ as required for a pure commission scheme.
} 
As in the basic model, the optimal incentive scheme is not unique. However, as the proof of corollary 2 shows, the commission is the only incentive scheme that induces $e_{1}=e_{2}=1$ and is independent of $\beta .{ }^{15}$ Hence, if the firm always wants to induce high effort, it can implement a commission even without knowing $\beta$. This is relevant because in practice the firm will often have less precise information about $\beta$ than the agent.

We now consider the case where markets are rather thin. In this case, another effort profile can be optimal. Define

$$
\bar{\Omega}\left(\beta_{0}, \beta_{1}\right):=\min \left\{\frac{1-(1-\alpha) \beta_{0}+(\rho-\alpha) \beta_{1}}{1-\beta_{0}+(\alpha+\rho) \beta_{1}}, \frac{1-(\rho-\alpha) \beta_{0}+(\rho-\alpha) \beta_{1}}{1-(\alpha+\rho) \beta_{0}+(\alpha+\rho) \beta_{1}}\right\} \cdot \frac{\alpha+\rho}{\rho^{2}} .
$$

Proposition 3 Assume that the market is rather thin, i.e., $\beta_{0}>2 \beta_{1}$. If $(\alpha+\rho) / \rho^{2}<\frac{R}{c}<$ $\bar{\Omega}\left(\beta_{0}, \beta_{1}\right)$, the firm induces high effort only in the second period and implements the compensation plan $w_{d u}^{F C}=\left(0,0, \frac{c}{\rho}, \frac{c}{\rho}\right)$. Otherwise, the cases (i)-(iii) in Proposition 2 apply.

Proof. See the proof of Proposition 2 in the Appendix.

When the firm faces a very thin market and the revenue-cost ratio is rather small, it is not optimal for the firm to induce high effort in the first period. The reason is the impact of the strong negative externality of high first-period effort on second-period demand. The firm thus offers a fixed salary for the first period and focuses on the provision of second-period incentives, paying the agent the commission $c / \rho$ independent of his first-period performance.

\section{Preference Uncertainty}

In this section, we drop the assumption of the basic model that the firm and the agent exactly know how exerting high effort affects the probability of a sale. This simplifying assumption seems realistic if the firm and the agents know the customers' preferences very well and can thus anticipate how the customers respond to high effort. However, there also exist situations in which the market's characteristics are not perfectly known. In particular, how customers will respond to effort might not be predictable. For example, when launching a new product or entering a foreign market with a given product, the firm and its agents do not exactly know whether it will be easy (i.e., high value of $\rho$ ) or difficult (i.e., low value of $\rho$ ) to sell the product.

To analyze such preference uncertainty, we assume that the market either consists of type-1 customers being characterized by $\rho=\rho_{1}$, or it consists of type- 2 customers being described by

\footnotetext{
${ }^{15}$ There is no incentive scheme that is equivalent to the quota $w_{d u}^{Q}$ but independent of $\beta$.
} 
$\rho=\rho_{2}$. The customer type is distributed such that

$$
\rho= \begin{cases}\rho_{1} & \text { with probability } p \\ \rho_{2} & \text { with probability } 1-p\end{cases}
$$

with $\rho_{t}+\alpha<1(t=1,2)$. Hence, we also have $E[\rho]+\alpha<1$ with $E[\rho]=p \rho_{1}+(1-p) \rho_{2}$. The corresponding variance is given by $\operatorname{Var}[\rho]=\left(\rho_{1}-E[\rho]\right)^{2} p+\left(\rho_{2}-E[\rho]\right)^{2}(1-p)$. As in the basic model, we assume that exerting high effort is efficient, i.e., $E[\rho] R-c>0$. We further assume that observed output in period 1 is not a reliable signal to update beliefs over $\rho$ (e.g., because the market is large so that observing one customer's behavior is a negligible sample). Hence, the probability distribution of $\rho$ remains the same for the two periods. All remaining assumptions of the basic model (e.g., a second customer that arrives for sure in period 2) are retained. We obtain the following result:

Proposition 4 The optimal sales force compensation is unique. If $R / c$ is sufficiently small, the firm will choose the fixed salary $w^{F}$. If $R / c$ is sufficiently large, the firm will either use the cutoff incentive plan $w_{p u}^{Q}=(0,0,0, c(1+E[\rho]) / E[\rho(\alpha+\rho)])$ or the permanent incentive plan $w_{p u}^{L}=(0,0, c / E[\rho],(c / E[\rho])+(c / E[\rho(\alpha+\rho)]))$. While $w_{p u}^{Q}$ is a simple quota-based bonus, $w_{p u}^{L}$ reflects a long-term bonus scheme that emphasizes second-period success. The firm prefers $w_{p u}^{Q}$ to $w_{p u}^{L}$ if and only if

$$
\left(\frac{E\left[(\alpha+\rho)^{2}\right]}{E[\rho(\alpha+\rho)]} E[\rho]-\frac{E[\alpha+\rho]}{E[\rho]}\right) c<\left(E\left[\rho^{2}\right]-(1-\alpha) E[\rho]\right) R
$$

\section{Proof. See Appendix.}

In contrast to the basic model and the case of demand uncertainty, preference uncertainty always leads to a unique solution for the optimal incentive contract. As in the basic model, the firm induces zero incentives if the revenue-cost ratio, $R / c$, is small. Otherwise, it either uses a quota-based bonus or a long-term bonus scheme. The latter leads to a positive payment only if the sales agent is successful with the second customer and rewards a first-period sale solely if it is followed by a second-period sale. Hence, the optimal incentive schemes emphasize long-term sales because they lead to zero payments in case of second-period failure. Rewarding short-term success by paying a commission per sale, which belongs to the optimal forms of sales force compensation in the basic model, is no longer optimal under preference uncertainty.

The proof of Proposition 4 gives a technical intuition for this result. The proof shows that, contrary to the basic model, the firm's iso-cost curves for implementing effort are flatter than 
the straight line describing the binding first-period incentive constraint. ${ }^{16}$ In other words, the introduction of preference uncertainty changes the rate of substitution between $w_{11}$ and $w_{10}$ in favor of $w_{11}$ in the firm's objective function. It is thus less costly for the firm to implement high effort by using $w_{11}$ instead of $w_{10}$. This leads to a unique solution for the optimal sales force compensation, which focuses on incentives for second-period sales.

Inspection of (13) shows under which conditions the firm prefers the quota-based bonus to the long-term bonus scheme and vice versa. The left-hand side of (13) is negative (see the Appendix for a proof). Hence, a sufficient condition for (13) to be satisfied is that the righthand side of (13) is positive. The right-hand side is increasing in $\alpha$ and, for $\alpha \rightarrow 1-E[\rho]$, it approaches

$$
\left(E\left[\rho^{2}\right]-(1-(1-E[\rho])) E[\rho]\right) R=\left(E\left[\rho^{2}\right]-E[\rho]^{2}\right) R=\operatorname{Var}[\rho] R,
$$

which is strictly positive. Thus, there exists a cut-off value $\bar{\alpha} \in(0,1-E[\rho])$ such that condition (13) is satisfied if $\alpha>\bar{\alpha}$. Recall that in the basic model the quota-based bonus is also attractive for the firm when $\alpha$ is large. Then a sale is rather likely to occur in the first period, implying that the disadvantage of the quota-based bonus - low effort in the second period after failure in the first - is unlikely to take effect. Hence, our findings on the optimality of the quota scheme from the basic model are robust with respect to the impact of $\alpha$.

The findings of this section indicate that bonus schemes are particularly effective incentive devices when firms launch new products or enter new markets. This result is in line with empirical observations. As Joseph and Kalwani (1998, p. 151) report, $26 \%$ of all bonus-paying firms use bonuses to boost incentives for selling new products. The authors conclude that "bonus payments tied to specific organizational goals such as promoting new product sales or sales to new customer groups help bring about goal congruence between the interests of the salesperson and the long-term objectives of the firm" (Joseph and Kalwani (1998), p. 158).

\section{Discussion}

In Sections 4 and 5, we introduced two alternative forms of uncertainty, resulting in a more comprehensive setup for the discussion of optimal sales force compensation. In the following, we will consider less fundamental extensions of the basic model to further test the robustness of our results.

\footnotetext{
${ }^{16}$ In the basic model, $\operatorname{Var}[\rho]=0$ so that both slopes are identical (see (32), (34) and (35)).
} 


\subsection{Non-Observability of the Sales Sequence}

So far we have assumed that the firm can costlessly observe sales at the end of each period. In practice, however, measuring sales performance is usually costly. Thus, ceteris paribus, the firm prefers to collect sales figures less frequently. To analyze the impact of scarcer sales information on optimal contracting, we now assume that the firm only observes total sales $x$ at the end of the second period, but not sales in each period $i, x_{i}$. This implies that the firm does not know the sequence of sales, i.e., when only one sale occured, the firm cannot tell whether the agent was successful in the first or second period. By contrast, the agent still observes sales in every period because he knows whether a customer bought the product after finishing the sales talk. In such a situation, the firm cannot base payments on the sequence of sales. Given our previous analysis, solving the firm's new problem is straightforward. We just have to add the restriction $w_{01}=w_{10}$ to the firm's problem in each scenario of the basic model from Section 3. Since the fixed wage $w^{F}$, the quota-based bonus $w^{Q}$, and the commission $w^{C}$ described in Proposition 1 all satisfy this condition, they are still feasible and hence optimal when the firm cannot observe the sales sequence. Moreover, the quota-based bonus and the commission are the only schemes from the previously optimal class of cutoff and permanent incentive plans, respectively, that satisfy $w_{01}=w_{10}$. Thus, scarcer sales information does not reduce the firm's profit but implies unique optimality of the two practically relevant incentive schemes.

Proposition 5 Suppose the firm cannot observe the sales sequence and thus $w_{01}=w_{10}$ has to hold. The firm then still earns the same profits as in the basic model. The optimal compensation plan, however, is unique: The firm either implements the fixed salary $w^{F}$ (if $\frac{R}{c} \leq \Omega_{L}(\alpha, \rho)$ ); or the quota-based bonus $w^{Q}$ (if $\Omega_{L}(\alpha, \rho)<\frac{R}{c}<\Omega_{H}(\alpha, \rho)$ ); or the commission $w^{C}$ (if $\Omega_{H}(\alpha, \rho) \leq$ $\left.\frac{R}{c}\right)$.

Besides pure bonuses and commissions, the third incentive scheme of high practical relevance is a combination of the two. It turns out that this incentive scheme, which belongs to the optimal class of cutoff incentive plans under demand uncertainty, is also uniquely optimal when the firm cannot observe the sales sequence.

Proposition 6 Suppose there is demand uncertainty as modeled in Section 4 and the firm cannot observe the sales sequence. Consider the case where markets are thin but not too thin, i.e., $\beta_{1} \leq \beta_{0} \leq 2 \beta_{1}$. The firm then still earns the same profits as under an observable sales sequence, but the optimal compensation plan is unique: The firm either implements the fixed salary $w^{F}$ (if $\frac{R}{c} \leq \hat{\Omega}_{L}$ ); or the quota-based bonus $w_{d u}^{Q}$ (if $\hat{\Omega}_{L}<\frac{R}{c} \leq \Omega_{H}(\alpha, \rho)$ ); or the combination of commission and bonus, $w_{d u}^{B C}$ (if $\left.\Omega_{H}(\alpha, \rho)<\frac{R}{c}\right)$. 


\subsection{Renegotiation-Proofness}

So far we have implicitly assumed that the firm can commit to a long-term contract covering both periods. However, if such commitment is not possible, both the firm and the agent may prefer to renegotiate the contract after having observed the first-period outcome. In that case, the contract would not be renegotiation-proof. In this section, we assume that the firm cannot commit to a long-term contract and hence has to derive the optimal renegotiation-proof contract. A contract covering both periods is renegotiation-proof if, at the beginning of the second period, there does not exist a short-term contract for period 2 that makes no party worse off but at least one party strictly better off compared to the given two-period contract.

First, consider the firm's optimal behavior in the second period, assuming for the moment that it is not restricted by any contract. The firm prefers either $e_{2}=1$ or $e_{2}=0$. To induce high effort $e_{2}=1$, the firm optimally offers the wage $c / \rho$ in case of success and zero payment in case of failure, leading to the profit $(\alpha+\rho)\left(R-\frac{c}{\rho}\right)$. Low effort $e_{2}=0$ is optimally implemented by a fixed wage of zero and hence the firm's profit is $\alpha R$. The firm thus prefers high effort to low effort if and only if $\frac{R}{c}>\frac{\alpha+\rho}{\rho^{2}}$. Note that $\frac{\alpha+\rho}{\rho^{2}} \in\left(\Omega_{L}(\alpha, \rho), \Omega_{H}(\alpha, \rho)\right)$, where $\Omega_{L}(\alpha, \rho)$ and $\Omega_{H}(\alpha, \rho)$ denote the two cut-off values from Proposition 1 .

We can now solve for the optimal renegotiation-proof contract. If $\frac{R}{c}>\Omega_{H}(\alpha, \rho)$, the firm again maximizes profits by implementing the commission scheme $w^{C}$. Because the commission induces high effort in both periods by paying $c / \rho$ per sale, the firm does not want to renegotiate the contract after the first period. If $\frac{R}{c} \in\left(\frac{\alpha+\rho}{\rho^{2}}, \Omega_{H}(\alpha, \rho)\right)$, the firm will no longer implement the quota scheme $w^{Q}$ or any other cutoff incentive plan because it is not renegotiation-proof. To see this, suppose the firm offered a cutoff incentive plan. If the agent fails in period 1 , he will exert zero effort in period 2 because he has no chance to realize two sales now. Hence, as $\frac{R}{c}>\frac{\alpha+\rho}{\rho^{2}}$, the firm would prefer to renegotiate the old contract and replace it by a short-term contract that offers wage $c / \rho$ in case of success and zero payment in case of a failure. The agent would accept this contract because he would then earn a rent. Thus, because in the given situation the firm always wants to induce high effort, the commission scheme $w^{C}$ is an optimal renegotiation-proof incentive scheme. If $\frac{R}{c} \in\left(\Omega_{L}(\alpha, \rho), \frac{\alpha+\rho}{\rho^{2}}\right]$, however, the quota scheme $w^{Q}$ will be renegotiation-proof since the firm has no incentive to offer a new contract after a firstperiod failure. If $\frac{R}{c} \leq \Omega_{L}(\alpha, \rho)$, the firm is always interested in inducing zero effort. Hence, the fixed salary $w^{F}$ is still optimal. The following proposition summarizes our findings:

Proposition 7 Suppose the firm cannot commit to a two-period long-term contract so that optimal contracts have to be renegotiation-proof. If $\frac{R}{c} \leq \Omega_{L}(\alpha, \rho)$, the fixed salary $w^{F}$ is optimal. If $\frac{R}{c} \in\left(\Omega_{L}(\alpha, \rho), \frac{\alpha+\rho}{\rho^{2}}\right]$, the quota-based bonus $w^{Q}$ is optimal. If $\frac{R}{c}>\frac{\alpha+\rho}{\rho^{2}}$, the commission 
scheme $w^{C}$ is optimal.

A comparison of Proposition 1 and Proposition 7 shows that, compared to the basic model, the firm should replace a quota scheme with a commission for a certain set of parameters if it cannot commit to long-term contracts. Intuitively, if the revenue-cost ratio is sufficiently large, the firm is always interested in inducing high effort. Thus, maintaining the quota scheme $w^{Q}$ in case of first-period failure is then not credible to the agent. Such a problem does not occur under a commission or a fixed salary where incentives remain constant over time. Note that the credibility problem of the quota-based bonus is also detrimental to first-period incentives. Anticipating renegotiation of the quota scheme in case of first-period failure, ${ }^{17}$ the agent will exert high effort in period 1 if and only if

$$
\begin{gathered}
(\alpha+\rho)\left[(\alpha+\rho) w_{11}^{Q}+(1-\alpha-\rho) w_{10}^{Q}-c\right]+(1-\alpha-\rho)\left[(\alpha+\rho) w_{01}^{r}+(1-\alpha-\rho) w_{00}^{r}\right]-c \\
\geq \alpha\left[(\alpha+\rho) w_{11}^{Q}+(1-\alpha-\rho) w_{10}^{Q}-c\right]+(1-\alpha)\left[(\alpha+\rho) w_{01}^{r}+(1-\alpha-\rho) w_{00}^{r}\right] \\
\Leftrightarrow \rho\left[(\alpha+\rho) w_{11}^{Q}+(1-\alpha-\rho) w_{10}^{Q}-(\alpha+\rho) w_{01}^{r}-(1-\alpha-r) w_{00}^{r}\right] \geq(1+\rho) c,
\end{gathered}
$$

where superscript $r$ denotes the payments after renegotiation of the quota scheme, i.e., $w_{01}^{r}=\frac{c}{\rho}$ and $w_{00}^{r}=0$. It is easily verified that the condition does not hold. Thus, first period incentives are completely erased.

Negative incentive effects from renegotiating initial contracts are also known from other long-term incentive schemes such as stock options. ${ }^{18}$ Such schemes will become ineffective when a manager learns that it is impossible for him to reach the long-term goal. If the manager anticipates that the long-term scheme may be adjusted or complemented by an optimal shortterm scheme later on, even early incentives can be completely erased.

\subsection{Advertising}

Firms often invest in advertising to increase sales. Our findings from the basic model allow us to discuss the impact of advertising on the optimal sales force compensation. In principle, there are two possibilities how advertising and the agent's effort can interact to boost sales. Depending on the product's characteristics, effort and advertising can be either substitutes or complements. Consider, first, the case of effort and advertising being substitutes, i.e., exerting high effort is less important to sell the product with advertising than without. Advertising then increases the basic probability of a sale, $\alpha$. For example, this is the case if advertising

\footnotetext{
${ }^{17}$ The quota scheme would not be renegotiated after a first-period success because the agent would not agree to lowering $w_{11}$.

${ }^{18}$ See, e.g., Acharya et al. (2000) on the incentive effects of resetting strike prices on stock options.
} 
informs about a low product price and customers primarily decide on the basis of price when choosing between different products. Second, advertising and effort can be complements, i.e., sales become more responsive to effort under advertising. In this case, advertising increases $\rho$. For example, consider the case of highly complex products. Advertising can be used to call the customers' attention to new innovative features of these products, making the customers curious. But sales effort is still needed to explain to them the usefulness of the new features. In this situation, the advertised features help the agent during his sales talk.

Because both $\Omega_{L}(\alpha, \rho)$ and $\Omega_{H}(\alpha, \rho)$ increase in $\alpha$, advertising reduces the provision of incentives and hence effort when advertising and effort are substitutes. This result nicely corresponds to the observation by Basu et al. (1985, p. 269) that salaries are typical of industries like the packaged goods industries where advertising already strongly affects sales. By contrast, $\Omega_{L}(\alpha, \rho)$ decreases in $\rho$, implying that a fixed salary becomes less likely under advertising when effort and advertising are complements. Despite this complementarity, however, advertising may actually lead to lower effort because the effect of $\rho$ on $\Omega_{H}(\alpha, \rho)$ is ambiguous (comp. Fig. 1): if $\rho$ is already quite large and is further increased by advertising, the firm will replace a permanent incentive plan (e.g., commission) with a cutoff incentive plan (e.g., quota-based bonus).

\subsection{Limited Liability of the Firm}

In our basic model, only the sales agent is protected by limited liability or, equivalently, is wealth-constrained. However, the firm may be wealth-constrained as well, which can make large incentive payments to the agent infeasible. Suppose the firm has zero initial wealth so that wage payments to the agent cannot exceed total revenues $x R$ with $x \in\{0,1,2\}$. In that case, the optimal fixed salary in scenario 1 is clearly feasible. The optimal commission in scenario 2 is also feasible because $\frac{c}{\rho} \leq R$ is implied by the assumption $\rho R-c \geq 0$. By contrast, the optimal quota scheme in scenario 3 may not be applicable any longer since the wage $w_{11}=\frac{1+\rho}{\alpha+\rho} \frac{c}{\rho}$ can be larger than $2 R$ for $\Omega_{L}(\alpha, \rho)<\frac{R}{c}<\Omega_{H}(\alpha, \rho){ }^{19}$

Proposition 8 If the firm has zero initial wealth, the fixed salary and the commission are still feasible, but the quota-based bonus is feasible if and only if $\frac{1+\rho}{2 \rho(\alpha+\rho)}<\frac{R}{c}$.

The intuition is the following. When an agent is risk neutral but protected by limited liability, the optimal quota scheme typically combines a large quota with a high bonus in case

\footnotetext{
${ }^{19}$ Note that $w_{11}<2 R$ is equivalent to $\frac{1+\rho}{2 \rho(\alpha+\rho)}<\frac{R}{c}$. Further, we have $\frac{1+\rho}{2 \rho(\alpha+\rho)} \in\left(\Omega_{L}(\alpha, \rho), \Omega_{H}(\alpha, \rho)\right)$ if, e.g., $\alpha=0.1$ and $\rho=0.5$.
} 
of success. ${ }^{20}$ In our setting, the sales agent will only obtain a positive bonus if he is successful in both periods, which yields the maximum possible quota. The corresponding bonus payment $w_{11}$ can be so large that it exceeds total revenues $2 R$. The condition in Proposition 8 shows that a wealth-constrained firm can apply a quota-based bonus when $\alpha$ is sufficiently large because then the bonus $w_{11}$ is rather small. ${ }^{21}$ If the condition does not hold, the firm will still employ a cutoff incentive plan when $\Omega_{L}(\alpha, \rho)<\frac{R}{c}<\Omega_{H}(\alpha, \rho)$. The firm then maximizes its profit by the incentive scheme $\left(0,(1-\alpha) \frac{c}{\rho}, 0,(2-\alpha) \frac{c}{\rho}\right)$, which satisfies the conditions in (10) and never exceeds total revenues.

\section{Conclusions}

We analyze a dynamic moral-hazard model on optimal sales force compensation. Inducing incentives is costly for the firm since sales agents earn rents. Our theoretical framework allows to design the optimal compensation plan without imposing any ad hoc restrictions on the class of feasible contracts. Frequently observed incentive plans - quota-based bonuses, commissions, and a combination thereof - turn out to belong to the optimal contract classes, but are mutually exclusive. It is therefore crucial for the firm to accurately investigate its specific environment before deciding on sales force incentives. We show that the aforementioned incentive plans can even be the unique solution to the firm's optimization problem when there is asymmetric information between the agent and the firm regarding the degree of demand uncertainty or the sequence of sales.

Our analysis leads to several testable predictions. First, firms with higher revenues are more likely to adapt incentive plans. Moreover, as revenue increase, firms should favor commissions over quota-based bonuses. For given revenues, firms with rather low or rather high effortsales responsiveness ${ }^{22}$ should prefer bonuses to commissions. If there is demand uncertainty, firms with intermediate revenues continue to rely on quota-based bonuses. High-revenue firms, however, are likely to adopt more sophisticated compensation plans. If markets are thin but not too thin, such firms should supplement a commission by a quota-based bonus. If present sales increase future sales prospects (e.g., through word-of-mouth advertising), dynamic commissions that increase over time should be observed. By contrast in very thin markets firms should use incentives cautiously, e.g., commission plans should be observed only toward the end of the product-life cycle. If a high-revenue firm enters a new market or launches a new product, it is

\footnotetext{
${ }^{20}$ See also Demougin and Fluet (1998) and Oyer (2000) on a model with continuous effort.

${ }^{21}$ Note that $\lim _{\alpha \rightarrow 1-\rho} \frac{1+\rho}{2 \rho(\alpha+\rho)}=\frac{1+\rho}{2 \rho}<\frac{1}{\rho}$, which together with the assumption $\frac{1}{\rho}<\frac{R}{c}$ implies that the condition in Proposition 5 holds for sufficiently large $\alpha$.

${ }^{22}$ Effort-sales responsiveness can be approximated by sales response functions.
} 
likely to rely on long-term incentive schemes that particularly emphasize success in later periods. Low-revenue firms should still employ simple quota-based bonuses. Finally, commissions tend to be more prevalent than quota-based bonuses in firms that face a tight liquidity condition, cannot commit to long-term compensation plans, or find it hard to predict future demand developments.

To keep the analysis tractable, we investigate dynamic aspects of sales force compensation plans in a two-period framework. This is the simplest way to capture that intermediate information on sales performance can induce the agent to adapt his effort. In practice, intermediate information may be available more often during the contract period. However, one also has to keep in mind that contract duration is restricted by limited commitment to long-term contracting and the fact that the firm cannot postpone payments to agents for too long. A general multiperiod framework leads to a considerably larger set of feasible effort allocations and, thus, candidate solutions to the firm's contracting problem. Nevertheless, within our basic framework, a pure commission still belongs to the optimal class of permanent incentive plans that always induce high effort. Furthermore, with a larger variety in design, we also expect quota-based bonus schemes to remain optimal cutoff incentive plans. Under demand uncertainty, a combination of commission and quota scheme should still be optimal under certain circumstances.

\section{Appendix}

\section{Proof of Proposition 1:}

The commission scheme (scenario 2) will dominate the quota scheme (scenario 3) iff

$$
\begin{gathered}
2(\alpha+\rho)\left(R-\frac{c}{\rho}\right)>R[(2+\rho) \alpha+\rho(1+\rho)]-(\alpha+\rho)(1+\rho) \frac{c}{\rho} \\
\Leftrightarrow \frac{R}{c}>\frac{1-\rho}{1-(\alpha+\rho)} \frac{\alpha+\rho}{\rho^{2}}=: \Omega_{H}(\alpha, \rho) .
\end{gathered}
$$

The commission will outperform the fixed salary (scenario 1) iff

$$
2(\alpha+\rho)\left(R-\frac{c}{\rho}\right)>2 \alpha R \Leftrightarrow \frac{R}{c}>\frac{\alpha+\rho}{\rho^{2}} .
$$

Note that $\Omega_{H}(\alpha, \rho)>\frac{\alpha+\rho}{\rho^{2}}$. Hence, the commission is the optimal incentive scheme if and only if $\frac{R}{c}>\Omega_{H}(\alpha, \rho)$. Otherwise, the firm prefers a quota-based bonus to the fixed wage if and only 


$$
R[(2+\rho) \alpha+\rho(1+\rho)]-(\alpha+\rho)(1+\rho) \frac{c}{\rho}>2 \alpha R \Leftrightarrow \frac{R}{c}>\frac{1+\rho}{1+\alpha+\rho} \frac{\alpha+\rho}{\rho^{2}}=: \Omega_{L}(\alpha, \rho) .
$$

Proof of Proposition 2:

There are four candidate solutions for the optimal incentive contract, whereas the remaining possible contracts can never be optimal (see the Additional Material 2).

(I) If the firm wants to induce $e_{1}=e_{2}=0$, it will offer $w^{F}$ and obtains expected profits

$$
\left[1+\alpha \beta_{1}+(1-\alpha) \beta_{0}\right] \alpha R
$$

(II) Next, suppose the firm wishes to induce $e_{1}=e_{2}=1$. If the agent has met the second customer, the second-period incentive constraint (2) from the basic model will apply again. However, the first-period incentive constraint under demand uncertainty now reads as follows:

$$
\begin{gathered}
(\alpha+\rho)\left[\beta_{1}\left((\alpha+\rho) w_{11}+(1-\alpha-\rho) w_{10}-c\right)+\left(1-\beta_{1}\right) w_{10}\right] \\
+(1-\alpha-\rho)\left[\beta_{0}\left((\alpha+\rho) w_{01}+(1-\alpha-\rho) w_{00}-c\right)+\left(1-\beta_{0}\right) w_{00}\right]-c \\
\geq \alpha\left[\beta_{1}\left((\alpha+\rho) w_{11}+(1-\alpha-\rho) w_{10}-c\right)+\left(1-\beta_{1}\right) w_{10}\right] \\
+(1-\alpha)\left[\beta_{0}\left((\alpha+\rho) w_{01}+(1-\alpha-\rho) w_{00}-c\right)+\left(1-\beta_{0}\right) w_{00}\right] \\
\Leftrightarrow(\alpha+\rho)\left(\beta_{1} w_{11}-\beta_{0} w_{01}\right)+(1-\alpha-\rho)\left(\beta_{1} w_{10}-\beta_{0} w_{00}\right) \\
+\left(1-\beta_{1}\right) w_{10}-\left(1-\beta_{0}\right) w_{00} \geq\left[1-\rho\left(\beta_{0}-\beta_{1}\right)\right] \frac{c}{\rho} .
\end{gathered}
$$

Hence, the firm solves

$$
\begin{aligned}
& \min _{w_{11}, w_{10}, w_{01}, w_{00} \geq 0}(\alpha+\rho)\left[\beta_{1}\left((\alpha+\rho) w_{11}+(1-\alpha-\rho) w_{10}\right)+\left(1-\beta_{1}\right) w_{10}\right] \\
& +(1-\alpha-\rho)\left[\beta_{0}\left((\alpha+\rho) w_{01}+(1-\alpha-\rho) w_{00}\right)+\left(1-\beta_{0}\right) w_{00}\right]
\end{aligned}
$$

subject to (2) and (18), leading to optimal payments $w_{00}=0$ and $w_{01}=\frac{c}{\rho}$. The firm's problem can, therefore, be simplified to

$$
\min _{w_{11}, w_{10} \geq 0}(\alpha+\rho)\left[\beta_{1}\left((\alpha+\rho) w_{11}+(1-\alpha-\rho) w_{10}\right)+\left(1-\beta_{1}\right) w_{10}+(1-\alpha-\rho) \beta_{0} \frac{c}{\rho}\right]
$$


subject to $w_{11} \geq \frac{c}{\rho}+w_{10}$ and

$$
\beta_{1}\left((\alpha+\rho) w_{11}+(1-\alpha-\rho) w_{10}\right)+\left(1-\beta_{1}\right) w_{10} \geq\left[1-\rho\left(\beta_{0}-\beta_{1}\right)+(\alpha+\rho) \beta_{0}\right] \frac{c}{\rho} .
$$

The firm thus optimally chooses wages $\left(w_{10}, w_{11}\right)$ that satisfy

$$
\beta_{1}\left((\alpha+\rho) w_{11}+(1-\alpha-\rho) w_{10}\right)+\left(1-\beta_{1}\right) w_{10}=\left[1-\rho\left(\beta_{0}-\beta_{1}\right)+(\alpha+\rho) \beta_{0}\right] \frac{c}{\rho}
$$

and $w_{11} \geq \frac{c}{\rho}+w_{10}$ and $w_{10} \geq 0$. The set of optimal contracts can also be rewritten as

$$
w_{11}=\frac{1-\rho\left(\beta_{0}-\beta_{1}\right)+(\alpha+\rho) \beta_{0}}{\beta_{1}(\alpha+\rho)} \frac{c}{\rho}-\frac{1-\beta_{1}(\alpha+\rho)}{\beta_{1}(\alpha+\rho)} w_{10} .
$$

and $0 \leq w_{10} \leq\left[1+\alpha\left(\beta_{0}-\beta_{1}\right)\right] \frac{c}{\rho}$. Consequently, if $\beta_{0} \geq \beta_{1}$, the incentive scheme

$$
\left(0, \frac{c}{\rho}, \frac{c}{\rho}, \frac{\alpha \beta_{0}+(\alpha+2 \rho) \beta_{1}}{\beta_{1}(\alpha+\rho)} \frac{c}{\rho}\right)=\left(0, \frac{c}{\rho}, \frac{c}{\rho},\left[2+\frac{\alpha}{\alpha+\rho} \frac{\beta_{0}-\beta_{1}}{\beta_{1}}\right] \frac{c}{\rho}\right)
$$

implements high effort in both periods at minimal cost. If $\beta_{0}<\beta_{1}$, however, the firm must set $w_{10}<w_{01}$. The scheme $\left(0,\left[1+\alpha\left(\beta_{0}-\beta_{1}\right)\right] \frac{c}{\rho}, \frac{c}{\rho},\left[2+\alpha\left(\beta_{0}-\beta_{1}\right)\right] \frac{c}{\rho}\right)$ then minimizes wage costs. The firm's expected profit is

$$
(\alpha+\rho)\left\{\left[1+(\alpha+\rho) \beta_{1}+(1-\alpha-\rho) \beta_{0}\right] R-\left[1+(1-\rho) \beta_{0}+\rho \beta_{1}\right] \frac{c}{\rho}\right\}
$$

(III) Now suppose the firm induces $e_{1}=1$ but $e_{2}=1$ if and only if $x_{1}=1$. The first-period incentive constraint can be written as

$$
\begin{gathered}
(\alpha+\rho)\left[(\alpha+\rho) \beta_{1} w_{11}+\left(1-\beta_{1}(\alpha+\rho)\right) w_{10}-\beta_{1} c\right]+(1-\alpha-\rho)\left[\alpha \beta_{0} w_{01}+\left(1-\alpha \beta_{0}\right) w_{00}\right]-c \\
\quad \geq \alpha\left[(\alpha+\rho) \beta_{1} w_{11}+\left(1-\beta_{1}(\alpha+\rho)\right) w_{10}-\beta_{1} c\right]+(1-\alpha)\left[\alpha \beta_{0} w_{01}+\left(1-\alpha \beta_{0}\right) w_{00}\right] \\
\quad \Leftrightarrow(\alpha+\rho) \beta_{1} w_{11}+\left(1-\beta_{1}(\alpha+\rho)\right) w_{10}-\alpha \beta_{0} w_{01}-\left(1-\alpha \beta_{0}\right) w_{00} \geq \frac{\left(1+\rho \beta_{1}\right)}{\rho} c .
\end{gathered}
$$

The firm thus minimizes

$$
(\alpha+\rho)\left[\beta_{1}\left((\alpha+\rho) w_{11}+(1-\alpha-\rho) w_{10}\right)+\left(1-\beta_{1}\right) w_{10}\right]+(1-\alpha-\rho)\left[\alpha \beta_{0} w_{01}+\left(1-\alpha \beta_{0}\right) w_{00}\right]
$$

subject to $w_{11} \geq \frac{c}{\rho}+w_{10}, w_{01} \leq \frac{c}{\rho}+w_{00}$, and (23). Therefore, $w_{00}=w_{01}=0$, and the two 
other optimal wages are described by

$$
\min (\alpha+\rho)\left[\beta_{1}\left((\alpha+\rho) w_{11}+(1-\alpha-\rho) w_{10}\right)+\left(1-\beta_{1}\right) w_{10}\right]
$$

subject to $w_{11} \geq \frac{c}{\rho}+w_{10}$ and (23). Under the optimal contract, the latter constraint boils down to

$$
(\alpha+\rho) \beta_{1} w_{11}+\left(1-\beta_{1}(\alpha+\rho)\right) w_{10}=\frac{\left(1+\rho \beta_{1}\right)}{\rho} c .
$$

Optimal are, e.g., $w_{10}=0$ and $w_{11}=\frac{1+\rho \beta_{1}}{(\alpha+\rho) \beta_{1}} \frac{c}{\rho}$, which - together with $w_{00}=w_{01}=0$ - describes a quota scheme. The firm's expected profit is

$$
(\alpha+\rho)\left[R+\beta_{1}(\alpha+\rho) R\right]+(1-\alpha-\rho) \beta_{0} \alpha R-(\alpha+\rho) \frac{\left(1+\rho \beta_{1}\right)}{\rho} c .
$$

(IV) Consider the case where effort is high only with customer 2: $e_{2}=1$ and $e_{1}=0$. The firm minimizes

$$
\begin{aligned}
& \alpha\left[\beta_{1}\left((\alpha+\rho) w_{11}+(1-(\alpha+\rho)) w_{10}\right)+\left(1-\beta_{1}\right) w_{10}\right] \\
& +(1-\alpha)\left[\beta_{0}\left((\alpha+\rho) w_{01}+(1-(\alpha+\rho)) w_{00}\right)+\left(1-\beta_{0}\right) w_{00}\right]
\end{aligned}
$$

subject to $w_{01} \geq \frac{c}{\rho}+w_{00}$ and $w_{11} \geq \frac{c}{\rho}+w_{10}$ as second-period incentive constraints, and

$$
\begin{gathered}
\alpha\left[\beta_{1}\left((\alpha+\rho) w_{11}+(1-(\alpha+\rho)) w_{10}-c\right)+\left(1-\beta_{1}\right) w_{10}\right] \\
+(1-\alpha)\left[\beta_{0}\left((\alpha+\rho) w_{01}+(1-(\alpha+\rho)) w_{00}-c\right)+\left(1-\beta_{0}\right) w_{00}\right] \\
\geq(\alpha+\rho)\left[\beta_{1}\left((\alpha+\rho) w_{11}+(1-(\alpha+\rho)) w_{10}-c\right)+\left(1-\beta_{1}\right) w_{10}\right] \\
+(1-(\alpha+\rho))\left[\beta_{0}\left((\alpha+\rho) w_{01}+(1-(\alpha+\rho)) w_{00}-c\right)+\left(1-\beta_{0}\right) w_{00}\right]-c \Leftrightarrow \\
\left(1+\rho\left(\beta_{1}-\beta_{0}\right)\right) \frac{c}{\rho} \geq \beta_{1}(\alpha+\rho) w_{11}+\left(1-(\alpha+\rho) \beta_{1}\right) w_{10}-\left(1-(\alpha+\rho) \beta_{0}\right) w_{00}-\beta_{0}(\alpha+\rho) w_{01}
\end{gathered}
$$

as first-period incentive constraint. Thus, $w_{00}=w_{10}=0$ and $w_{01}=w_{11}=\frac{c}{\rho}$ are optimal. This compensation leads to expected profit

$$
\left(\alpha+(1-\alpha)(\alpha+\rho) \beta_{0}+\alpha(\alpha+\rho) \beta_{1}\right) R-\left((1-\alpha) \beta_{0}+\alpha \beta_{1}\right)(\alpha+\rho) \frac{c}{\rho} .
$$

We now compare the profits in the different scenarios. Profit in scenario (IV) will be larger than profit in scenario (I) iff

$$
\frac{\alpha+\rho}{\rho^{2}}<\frac{R}{c}
$$


Moreover, profit in scenario (IV) exceeds the profits in scenarios (II) and (III), respectively, iff

$$
\frac{R}{c}<\frac{1-(\rho-\alpha) \beta_{0}+(\rho-\alpha) \beta_{1}}{1-(\alpha+\rho) \beta_{0}+(\alpha+\rho) \beta_{1}} \frac{\alpha+\rho}{\rho^{2}}
$$

and

$$
\frac{R}{c}<\frac{1-(1-\alpha) \beta_{0}+(\rho-\alpha) \beta_{1}}{1-\beta_{0}+(\alpha+\rho) \beta_{1}} \frac{\alpha+\rho}{\rho^{2}} .
$$

For the conditions (27)-(29) to hold at the same time, it is necessary that $\beta_{0}>2 \beta_{1}$, which guarantees that the right-hand sides of $(28)$ and (29) are larger than $\frac{\alpha+\rho}{\rho^{2}}$. If one of the three conditions (27)-(29) is violated, the firm will prefer one of the scenarios (I)-(III). Expected profits (22) in scenario (II) exceed expected profits (25) in scenario (III) iff condition (14) holds, i.e., iff $R / c>\Omega_{H}(\alpha, \rho)$. Comparing (17) and (25) yields that the fixed salary of scenario (I) leads to higher profits then the quota scheme of scenario (III) iff

$$
\frac{R}{c}<\frac{1+\rho \beta_{1}}{1-\alpha \beta_{0}+(2 \alpha+\rho) \beta_{1}} \frac{\alpha+\rho}{\rho^{2}}:=\hat{\Omega}_{L}<\Omega_{H}(\alpha, \rho)
$$

\section{Proof of Corollary 2:}

Because $\frac{R}{c}>\Omega_{H}(\alpha, \rho)$, the firm optimally induces $e_{1}=e_{2}=1$. By the proof of Proposition 2 , an optimal compensation scheme is described by $(20)$ and $0 \leq w_{10} \leq\left[1+\alpha\left(\beta_{0}-\beta_{1}\right)\right] \frac{c}{\rho}$. Inserting $\beta_{0}=\beta_{1}=\beta$ leads to $0 \leq w_{10} \leq \frac{c}{\rho}$ and

$$
\begin{aligned}
w_{11} & =\frac{1+(\alpha+\rho) \beta}{\beta(\alpha+\rho)} \frac{c}{\rho}-\frac{1-\beta(\alpha+\rho)}{\beta(\alpha+\rho)} w_{10} . \\
& =\frac{1+(\alpha+\rho) \beta}{\beta(\alpha+\rho)}-\frac{1-\beta(\alpha+\rho)}{\beta(\alpha+\rho)} \delta .
\end{aligned}
$$

Let $w_{10}=\delta \frac{c}{\rho}$ with $\delta \in[0,1]$. Thus, $w_{11}=\left(\frac{1-\delta}{\beta(\alpha+\rho)}+(1+\delta)\right) \frac{c}{\rho}$. For $\delta=1$, we obtain a commission scheme that is independent of $\beta$. For $\delta \in[0,1)$, the incentive scheme depends on $\beta$.

Now suppose $\hat{\Omega}_{L}<\frac{R}{c} \leq \Omega_{H}(\alpha, \rho)$ so that the firm induces $e_{1}=1$ but $e_{2}=1$ if and only if $x_{1}=1$. The corresponding optimal compensation is described by (24) and $w_{11} \geq \frac{c}{\rho}+w_{10}$ (see the proof of Proposition 2). Rewriting the two optimality conditions and using $\beta_{1}=\beta$ leads to

$$
w_{11}=\frac{1+\rho \beta}{\beta(\alpha+\rho)} \frac{c}{\rho}-\frac{1-\beta(\rho+\alpha)}{\beta(\alpha+\rho)} w_{10} \quad \text { and } \quad(1-\alpha \beta) \frac{c}{\rho} \geq w_{10}
$$

Assume $w_{10}=\delta(1-\alpha \beta) \frac{c}{\rho}, \delta \in[0,1]$. Thus, $w_{10}$ is independent of $\beta$ iff $\delta=0$. However, in this case, $w_{11}=\frac{1+\rho \beta}{\beta(\alpha+\rho)} \frac{c}{\rho}$ still depends on $\beta$, i.e., for the optimal incentive scheme the firm needs to 
know $\beta$.

\section{Proof of Proposition 4:}

(I) If the firm wants to induce zero incentives, it will offer $w^{F}=(0,0,0,0)$. Hence, the firm's expected profit is $2 \alpha R$.

(II) Suppose that the firm wants to induce $e_{1}=e_{2}=1$. To implement $e_{2}=1$ given the first-period outcome $x_{1} \in\{0,1\}$, it must hold that:

$$
\begin{gathered}
(\alpha+E[\rho]) w_{x_{1} 1}+(1-(\alpha+E[\rho])) w_{x_{1} 0}-c \geq \alpha w_{x_{1} 1}+(1-\alpha) w_{x_{1} 0} \\
\Leftrightarrow w_{x_{1} 1} \geq \frac{c}{E[\rho]}+w_{x_{1} 0}
\end{gathered}
$$

Analogously to the basic model, the first-stage incentive constraint reads as

$$
E\left[\rho\left((\alpha+\rho)\left(w_{11}-w_{01}\right)+(1-\alpha-\rho)\left(w_{10}-w_{00}\right)\right)\right] \geq c .
$$

The firm thus solves

$$
\min _{w_{11}, w_{10}, w_{01}, w_{00} \geq 0} E\left[(\alpha+\rho)^{2} w_{11}+(\alpha+\rho)(1-(\alpha+\rho))\left(w_{10}+w_{01}\right)+(1-(\alpha+\rho))^{2} w_{00}\right]
$$

$$
\text { s.t. }(30),(31) \text {. }
$$

The firm optimally chooses $w_{00}=0$ and $w_{01}=c / E[\rho]$. Concerning the optimal choice of $w_{11}$ and $w_{10}$, the firm minimizes total costs $C$ characterized by the iso-cost curves

$$
\frac{C}{A_{2}}-\frac{c}{E[\rho]}-\frac{A_{1}}{A_{2}} w_{11}=w_{10}
$$

where $A_{1}:=E\left[(\alpha+\rho)^{2}\right]$ and $A_{2}:=E[(\alpha+\rho)(1-(\alpha+\rho))]$, subject to the constraints

$$
\begin{aligned}
w_{11}-\frac{c}{E[\rho]} & \geq w_{10} \\
\text { and } \quad \frac{c}{A_{4}}+\frac{c}{E[\rho]} \frac{A_{3}}{A_{4}}-\frac{A_{3}}{A_{4}} w_{11} & \leq w_{10}
\end{aligned}
$$

with $A_{3}:=E[\rho(\alpha+\rho)]$ and $A_{4}:=E[\rho(1-(\alpha+\rho))]$. Graphically, the constraints (33) and (34) define a feasible region within the $\left(w_{11}, w_{10}\right)$-space, and the firm needs to identify the lowest isocost curve (32) that stays in the feasible region. Condition (33) corresponds to the area below a straight line with positive slope that intersects with the horizontal axis at $w_{11}=c / E[\rho]$. Condition (34) describes an area above a negatively sloped straight line that intersects with the horizontal axis at $w_{11}=(c / E[\rho])+\left(c / A_{3}\right)>c / E[\rho]$. Thus, the two straight lines intersect 
above the horizontal axis. Since the absolute value of the slope of the iso-cost curves is smaller than the absolute value of the slope of the straight line characterized by (34), i.e.,

$$
\frac{A_{1}}{A_{2}}<\frac{A_{3}}{A_{4}} \Leftrightarrow 0<\operatorname{Var}[\rho]
$$

the firm will optimally choose

$$
w_{11}=\frac{c}{E[\rho]}+\frac{c}{A_{3}}=\frac{c}{E[\rho]}+\frac{c}{E[\rho(\alpha+\rho)]} \quad \text { and } \quad w_{10}=0
$$

yielding expected profits

$$
\pi_{(I I)}=2 E[\alpha+\rho] R-\frac{E[\alpha+\rho]}{E[\rho]} c-\frac{E\left[(\alpha+\rho)^{2}\right]}{E[\rho(\alpha+\rho)]} c .
$$

(III) Suppose the firm induces $e_{1}=1$ but $e_{2}=1$ if and only if $x_{1}=1$. Analogous to the basic model, the first-stage incentive constraint is given by

$$
E[\rho(\alpha+\rho)]\left(w_{11}-w_{10}\right)-E[\rho \alpha]\left(w_{01}-w_{00}\right)+E[\rho]\left(w_{10}-w_{00}\right) \geq(1+E[\rho]) c .
$$

The firm thus minimizes

$$
E\left[(\alpha+\rho)^{2} w_{11}+(\alpha+\rho)(1-\alpha-\rho) w_{10}+(1-\alpha-\rho) \alpha w_{01}+(1-\alpha-\rho)(1-\alpha) w_{00}\right]
$$

subject to (36) and the second-period incentive constraints $w_{11} \geq \frac{c}{E[\rho]}+w_{10}$, and $w_{01}<\frac{c}{E[\rho]}+$ $w_{00}$. Hence, $w_{00}=w_{01}=0$ is optimal, and the firm minimizes costs $C$ characterized by the iso-cost curves

$$
\frac{C}{A_{2}}-\frac{A_{1}}{A_{2}} w_{11}=w_{10}
$$

subject to the constraints

$$
\begin{aligned}
w_{11}-\frac{c}{E[\rho]} & \geq w_{10} \\
\text { and } \quad \frac{(1+E[\rho]) c}{A_{4}}-\frac{A_{3}}{A_{4}} w_{11} & \leq w_{10} .
\end{aligned}
$$

Note that the zero of the straight line described by constraint (39) (i.e., $\left.w_{11}=(1+E[\rho]) \frac{c}{A_{3}}\right)$ is larger than the zero of the straight line described (38) $c / E[\rho]$ :

$$
(1+E[\rho]) \frac{c}{A_{3}}>\frac{c}{E[\rho]} \Leftrightarrow(1+E[\rho]) E[\rho]>E[\rho(\alpha+\rho)]
$$


is true since $E[\rho]>E[\rho(\alpha+\rho)] \Leftrightarrow p \rho_{1}+(1-p) \rho_{2}>p \rho_{1}\left(\alpha+\rho_{1}\right)+(1-p) \rho_{2}\left(\alpha+\rho_{2}\right)$ holds because $\rho_{t}+\alpha<1(t=1,2)$. Thus, we have qualitatively the same graphical solution as in case (II) above. The absolute value of the slope of the iso-cost curves is smaller than the absolute value of the slope of the of the straight line described by (39), i.e., condition (35) is satisfied. Hence, the firm optimally chooses

$$
w_{11}=(1+E[\rho]) \frac{c}{A_{3}}=(1+E[\rho]) \frac{c}{E[\rho(\alpha+\rho)]} \quad \text { and } \quad w_{10}=0
$$

which characterizes a quota-based bonus. Expected profits are

$$
\pi_{(I I I)}=E[\alpha+\rho] R+\left\{E\left[(\alpha+\rho)^{2}\right]+E[(1-\alpha-\rho) \alpha]\right\} R-\frac{E\left[(\alpha+\rho)^{2}\right](1+E[\rho]) c}{E[\rho(\alpha+\rho)]} .
$$

Besides the scenarios (I) to (III), there are four additional cases that can be implemented. From the firm's point of view, however, none of these cases is optimal (see the Additional Material $3)$.

The firm will prefer scenario (I) to scenario (II) iff

$$
\pi_{(I I)}<2 \alpha R \Leftrightarrow \frac{R}{c}<\frac{E[\alpha+\rho]}{2 E[\rho]^{2}}+\frac{E\left[(\alpha+\rho)^{2}\right]}{2 E[\rho] E[\rho(\alpha+\rho)]},
$$

and scenario (I) to scenario (III) iff

$$
\pi_{(I I I)}<2 \alpha R \Leftrightarrow \frac{R}{c}<\frac{E\left[(\alpha+\rho)^{2}\right](1+E[\rho])}{\left((1+\alpha) E[\rho]+E\left[\rho^{2}\right]\right) E[\rho(\alpha+\rho)]}
$$

It will prefer the quota scheme described by scenario (III) to the other compensation scheme described by (II) iff

$$
\pi_{(I I)}<\pi_{(I I I)} \Leftrightarrow\left(\frac{E\left[(\alpha+\rho)^{2}\right]}{E[\rho(\alpha+\rho)]} E[\rho]-\frac{E[\alpha+\rho]}{E[\rho]}\right) c<\left(E\left[\rho^{2}\right]-(1-\alpha) E[\rho]\right) R .
$$


Proof that the left-hand side of (13) is negative:

$$
\begin{gathered}
\frac{E\left[(\alpha+\rho)^{2}\right]}{E[\rho(\alpha+\rho)]} E[\rho]<\frac{E[\alpha+\rho]}{E[\rho]} \\
\Leftrightarrow \frac{\alpha^{2}+2 \alpha E[\rho]+E\left[\rho^{2}\right]}{\alpha E[\rho]+E\left[\rho^{2}\right]}<\frac{1}{E[\rho]}+\frac{\alpha}{E[\rho]^{2}} \\
\Leftrightarrow \alpha^{2}+2 \alpha E[\rho]+E\left[\rho^{2}\right]<\alpha+\frac{E\left[\rho^{2}\right]}{E[\rho]}+\frac{1}{E[\rho]} \alpha^{2}+\frac{E\left[\rho^{2}\right]}{E[\rho]^{2}} \alpha \\
\Leftrightarrow \frac{E[\rho]-1}{E[\rho]} \alpha^{2}+\frac{(2 E[\rho]-1) E[\rho]^{2}-E\left[\rho^{2}\right]}{E[\rho]^{2}} \alpha+\frac{E[\rho]-1}{E[\rho]} E\left[\rho^{2}\right]<0
\end{gathered}
$$

Dividing both sides by $\frac{E[\rho]-1}{E[\rho]}<0$ leads to

$$
\alpha^{2}+\frac{E\left[\rho^{2}\right]-(2 E[\rho]-1) E[\rho]^{2}}{(1-E[\rho]) E[\rho]} \alpha+E\left[\rho^{2}\right]>0
$$

Since $\operatorname{Var}[\rho]=E\left[\rho^{2}\right]-E[\rho]^{2}>0$ implies that $E\left[\rho^{2}\right]>E[\rho]^{2}$ and $2 E[\rho]-1<1 \Leftrightarrow E[\rho]<1$, condition (40) is true.

\section{References}

Acharya, V. V., K. John, and R. K. Sundaram (2000). On the optimality of resetting executive stock options. Journal of Financial Economics 57, 65-101.

Ackerberg, D. and M. Botticini (2002). Endogenous matching and the empirical determinants of contract form. Journal of Political Economy 110, 564-591.

Albers, S. and M. Mantrala (2008). Models for sales management decisions. In B. Wierenga (Ed.), Handbook of marketing decision models. Springer: Berlin.

Bak, B. and A. Klecz-Simon (2013). Quota bonuses with heterogeneous agents. Economics Letters 119, 316-320.

Basu, A., V. S. R. Lal, and R. Staelin (1985). Salesforce compensation plans: An agency theoretic perspective. Marketing Science 4, 267-291.

Bellemare, C. and B. Shearer (2010). Sorting, incentives and risk preferences: Evidence from a field experiment. Economics Letters 108, 345-348.

Bierbaum, J. (2002). Repeated moral hazard under limited liability. Working Paper.

Che, Y.-K. and S.-W. Yoo (2001). Optimal incentives for teams. American Economic Review 91, $525-541$. 
Chung, D. J., T. Steenburgh, and K. Sudhir (2013). Do bonuses enhance sales productivity? a dynamic structural analysis of bonus-based compensation plans. Harvard Business School Working Paper, No. 13-066.

Coughlan, A. T. (1993). Salesforce compensation: A review of MS/OR advances. In J. Eliashberg and G. L. Lilien (Eds.), Handbooks in Operations Research and Management Science, Marketing, Volume 5, pp. 611-651. Amsterdam: North-Holland.

Dearden, J. A. and G. L. Lilien (1990). On optimal salesforce compensation in the presence of production learning effects. International Journal of Research in Marketing 7, 179-188.

Demougin, D. and C. Fluet (1998). Mechanism sufficient statistic in the risk-neutral agency problem. Journal of Institutional and Theoretical Economics 154, 622-639.

Hilt, E. (2008). The negative trade-off between risk and incentives: Evidence from the american whaling industry. Explorations in Economic History 45, 424-444.

Holmstrom, B. and P. Milgrom (1987). Aggregation and linearity in the provision of intertemporal incentives. Econometrica 55, 303-328.

Innes, R. (1990). Limited liability and incentive contracting with ex-ante action choices. Journal of Economic Theory 52, 45-67.

Jain, S. (2012). Self-control and incentives: An analysis of multiperiod quota plans. Marketing Science 31, 855-869.

Joseph, K. and M. Kalwani (1998). The role of bonus pay in salesforce compensation plans. Industrial Marketing Management 27, 147-159.

Kaya, A. and G. Vereshchagina (2014). Partnerships versus corporations: Moral hazard, sorting, and ownership structure. American Economic Review 104, 291-307.

Kishore, S., R. Rao, O. Narasimhan, and G. John (2013). Bonuses versus commissions: A field study. Journal of Marketing Research 50, 317-333.

Laffont, J.-J. and D. Martimort (2002). The Theory of Incentives: The Principal-Agent Model. Princeton University Press: Princeton and Oxford.

Lal, R. and V. Srinivasan (1993). Compensation plans for single- and multi-product salesforces: An application of the Holmstrom-Milgrom model. Management Science 39, 777-793. 
Ohlendorf, S. and P. Schmitz (2012). Repeated moral hazard and contracts with memory: The case of risk-neutrality. International Economic Review 53, 433-452.

Oyer, P. (2000). A theory of sales quotas with limited liability and rent sharing. Journal of Labor Economics 18, 405-426.

Poblete, J. and D. Spulber (2012). The form of incentive contracts: Agency with moral hazard, risk neutrality, and limited liability. RAND Journal of Economics 43, 215-234.

Raju, J. S. and V. Srinivasan (1996). Quota-based compensation plans for multiterritory heterogeneous salesforces. Management Science 42, 1454-1462.

Sappington, D. (1983). Limited liability contracts between principal and agent. Journal of Economic Theory 29, 1-21.

Schmitz, P. (2005). Allocating control in agency problems with limited liability and sequential hidden actions. RAND Journal of Economics 36, 2, 318-336.

Schmitz, P. W. (2013). Job design with conflicting tasks reconsidered. European Economic Review 5\%, 108-117.

Simester, D. and J. Zhang (2010). Why are bad products so hard to kill? Management Science 56, 1161-1179.

Simester, D. and J. Zhang (2013). Why do sales people spend so much time lobbying for low prices? Working Paper.

Steenburgh, T. J. (2008). Effort or timing: The effect of lump-sum bonuses. Quantitative Marketing and Economics 6, 235-256.

Zoltners, A. A., P. Sinha, and S. E. Lorimer (2008). Sales force effectiveness: A framework for researchers and practitioners. Journal of Personal Selling and Sales Management 28, $115-131$. 


\section{Additional Material (not intended for publication)}

\section{Additional Material 1: The Suboptimal Cases in the Basic Model}

There exist four additional cases that can be chosen by the firm. First, consider the case where the firm induces $e_{1}=1$, but $e_{2}=1$ if and only if $x_{1}=0$. That is, the sales agent should exert high effort with the second customer only if he could not sell the product to the first customer. The first-stage incentive constraint thus becomes

$$
\begin{gathered}
(\alpha+\rho)\left[\alpha w_{11}+(1-\alpha) w_{10}\right]+(1-\alpha-\rho)\left[(\alpha+\rho) w_{01}+(1-\alpha-\rho) w_{00}-c\right]-c \\
\geq \alpha\left[\alpha w_{11}+(1-\alpha) w_{10}\right]+(1-\alpha)\left[(\alpha+\rho) w_{01}+(1-\alpha-\rho) w_{00}-c\right] \\
\Leftrightarrow \rho\left[\alpha w_{11}+(1-\alpha) w_{10}-(\alpha+\rho) w_{01}-(1-\alpha-\rho) w_{00}\right] \geq(1-\rho) c .
\end{gathered}
$$

The firm now solves

$$
\begin{aligned}
\min _{w_{11}, w_{10}, w_{01}, w_{00} \geq 0}(\alpha+\rho) \alpha w_{11}+(\alpha+\rho)(1-\alpha) w_{10}+(1-\alpha-\rho)(\alpha+\rho) w_{01}+(1-\alpha-\rho)^{2} w_{00} \\
\text { subject to } \quad w_{11}<\frac{c}{\rho}+w_{10}, \quad w_{01} \geq \frac{c}{\rho}+w_{00}, \quad \text { and }(41) .
\end{aligned}
$$

Consequently, $w_{00}=0$. Furthermore, $w_{01}$ should be as small as possible, $w_{01}=\frac{c}{\rho}$. It follows that $\alpha w_{11}+(1-\alpha) w_{10}$ should be minimal. Hence, by $(41)$ and $w_{11}<\frac{c}{\rho}+w_{10}$, all $w_{11}$ and $w_{10}$ satisfying

$$
\alpha w_{11}+(1-\alpha) w_{10}=\frac{1-\rho}{\rho} c+(\alpha+\rho) \frac{c}{\rho}=(1+\alpha) \frac{c}{\rho} \text { and } w_{11}<\frac{c}{\rho}+w_{10}
$$

are optimal (e.g., $w_{11}=0$ and $\left.w_{10}=\frac{1+\alpha}{1-\alpha} \frac{c}{\rho}\right)$. The firm's profit is

$$
\begin{aligned}
& (\alpha+\rho) R+[(\alpha+\rho) \alpha+(1-\alpha-\rho)(\alpha+\rho)] R-\left[(\alpha+\rho)(1+\alpha) \frac{c}{\rho}+(1-\alpha-\rho)(\alpha+\rho) \frac{c}{\rho}\right] \\
& =(2-\rho)(\alpha+\rho)\left(R-\frac{c}{\rho}\right)
\end{aligned}
$$

which is smaller than the profit in scenario 2 described by (6).

Second, consider the case where effort is high only with one customer: $e_{i}=1$ and $e_{j}=0$ $(i, j=1,2, i \neq j)$. Let, w.l.o.g., $i=1$. The firm minimizes

$$
(\alpha+\rho) \alpha w_{11}+(\alpha+\rho)(1-\alpha) w_{10}+(1-\alpha-\rho) \alpha w_{01}+(1-\alpha-\rho)(1-\alpha) w_{00}
$$


subject to $w_{01}<\frac{c}{\rho}+w_{00}$ and $w_{11}<\frac{c}{\rho}+w_{10}$ as second-stage incentive constraints, and

$$
\begin{gathered}
(\alpha+\rho)\left[\alpha w_{11}+(1-\alpha) w_{10}\right]+(1-\alpha-\rho)\left[\alpha w_{01}+(1-\alpha) w_{00}\right]-c \\
\geq \alpha\left[\alpha w_{11}+(1-\alpha) w_{10}\right]+(1-\alpha)\left[\alpha w_{01}+(1-\alpha) w_{00}\right] \\
\Leftrightarrow \alpha w_{11}+(1-\alpha) w_{10}-\alpha w_{01}-(1-\alpha) w_{00} \geq \frac{c}{\rho} .
\end{gathered}
$$

as first-stage incentive constraint. Thus, $w_{01}=w_{00}=0$, and optimal sales force compensation is described by $\alpha w_{11}+(1-\alpha) w_{10}=\frac{c}{\rho}$ and $w_{11}<\frac{c}{\rho}+w_{10}$, being satisfied, e.g., for $w_{11}=0$ and $w_{10}=\frac{1}{1-\alpha} \frac{c}{\rho}$. The profit is

$$
[2(\alpha+\rho) \alpha+(\alpha+\rho)(1-\alpha)+(1-\alpha-\rho) \alpha] R-(\alpha+\rho) \frac{c}{\rho}=[2 \alpha+\rho] R-(\alpha+\rho) \frac{c}{\rho} .
$$

This profit is smaller than profit (6) under scenario 2 if

$$
[2 \alpha+\rho] R-(\alpha+\rho) \frac{c}{\rho}<2(\alpha+\rho)\left(R-\frac{c}{\rho}\right) \Leftrightarrow \frac{R}{c}>\frac{\alpha+\rho}{\rho^{2}} .
$$

Profit (42) is smaller than the profit under scenario 1 if

$$
[2 \alpha+\rho] R-(\alpha+\rho) \frac{c}{\rho}<2 \alpha R \Leftrightarrow \frac{R}{c}<\frac{\alpha+\rho}{\rho^{2}} .
$$

Thus, inducing high effort only with one customer cannot be optimal.

Third, the firm may be interested in implementing low effort with customer 1 and high effort with customer 2 if and only if $x_{1}=1$, that is, $e_{1}=0$ and $e_{2}=1$ iff $x_{1}=1$. The first-stage incentive constraint is

$$
\begin{gathered}
\alpha\left[(\alpha+\rho) w_{11}+(1-\alpha-\rho) w_{10}-c\right]+(1-\alpha)\left[\alpha w_{01}+(1-\alpha) w_{00}\right] \\
\geq(\alpha+\rho)\left[(\alpha+\rho) w_{11}+(1-\alpha-\rho) w_{10}-c\right]+(1-\alpha-\rho)\left[\alpha w_{01}+(1-\alpha) w_{00}\right]-c \\
\Leftrightarrow(1+\rho) c \geq \rho\left[(\alpha+\rho) w_{11}+(1-\alpha-\rho) w_{10}-\alpha w_{01}-(1-\alpha) w_{00}\right] .
\end{gathered}
$$

Hence, the firm's problem can be characterized as follows:

$$
\begin{gathered}
\min _{w_{11}, w_{10}, w_{01}, w_{00} \geq 0} \alpha(\alpha+\rho) w_{11}+\alpha(1-\alpha-\rho) w_{10}+(1-\alpha) \alpha w_{01}+(1-\alpha)^{2} w_{00} \\
\text { subject to } \quad w_{01}<\frac{c}{\rho}+w_{00}, \quad w_{11} \geq \frac{c}{\rho}+w_{10}, \quad \text { and }(43) .
\end{gathered}
$$


It is optimal to have $w_{00}=w_{01}=w_{10}=0$ and $w_{11}=\frac{c}{\rho}$. Expected profit is

$$
\alpha R+\alpha(\alpha+\rho) R+(1-\alpha) \alpha R-\alpha(\alpha+\rho) \frac{c}{\rho}=(2+\rho) \alpha R-\alpha(\alpha+\rho) \frac{c}{\rho},
$$

which is smaller than (42) if

$$
(2+\rho) \alpha R-\alpha(\alpha+\rho) \frac{c}{\rho}<[2 \alpha+\rho] R-(\alpha+\rho) \frac{c}{\rho} \Leftrightarrow \frac{R}{c}>\frac{\alpha+\rho}{\rho^{2}} .
$$

On the other hand, (44) is smaller than profit under $e_{1}=e_{2}=0$ if

$$
(2+\rho) \alpha R-\alpha(\alpha+\rho) \frac{c}{\rho}<2 \alpha R \Leftrightarrow \frac{R}{c}<\frac{\alpha+\rho}{\rho^{2}},
$$

implying that this third alternative scenario cannot be optimal for the firm.

Fourth, the firm may wish to implement low effort with customer 1 and high effort with customer 2 if and only if $x_{1}=0$, that is, $e_{1}=0$ and $e_{2}=1$ iff $x_{1}=0$. The first-stage incentive constraint reads as

$$
\begin{gathered}
\alpha\left[\alpha w_{11}+(1-\alpha) w_{10}\right]+(1-\alpha)\left[(\alpha+\rho) w_{01}+(1-\alpha-\rho) w_{00}-c\right] \\
\geq(\alpha+\rho)\left[\alpha w_{11}+(1-\alpha) w_{10}\right]+(1-\alpha-\rho)\left[(\alpha+\rho) w_{01}+(1-\alpha-\rho) w_{00}-c\right]-c \\
\Leftrightarrow(1-\rho) c \geq \rho\left[\alpha w_{11}+(1-\alpha) w_{10}-(\alpha+\rho) w_{01}-(1-\alpha-\rho) w_{00}\right] .
\end{gathered}
$$

The firm's problem is

$$
\begin{array}{r}
\min _{w_{11}, w_{10}, w_{01}, w_{00} \geq 0} \alpha^{2} w_{11}+\alpha(1-\alpha) w_{10}+(1-\alpha)(\alpha+\rho) w_{01}+(1-\alpha)(1-\alpha-\rho) w_{00} \\
\text { subject to } w_{01} \geq \frac{c}{\rho}+w_{00}, \quad w_{11}<\frac{c}{\rho}+w_{10}, \quad \text { and }(45) .
\end{array}
$$

It is thus optimal to have $w_{00}=w_{10}=w_{11}=0$ and $w_{01}=\frac{c}{\rho}$. Expected profit is

$$
\alpha R+\alpha^{2} R+(1-\alpha)(\alpha+\rho) R-(1-\alpha)(\alpha+\rho) \frac{c}{\rho}=[2 \alpha+\rho-\alpha \rho] R-(1-\alpha)(\alpha+\rho) \frac{c}{\rho},
$$

which is smaller than profit (42) if

$$
[2 \alpha+\rho-\alpha \rho] R-(1-\alpha)(\alpha+\rho) \frac{c}{\rho}<[2 \alpha+\rho] R-(\alpha+\rho) \frac{c}{\rho} \Leftrightarrow \frac{R}{c}>\frac{\alpha+\rho}{\rho^{2}} .
$$


On the other hand, profit (46) is smaller than profit under $e_{1}=e_{2}=0$ if

$$
[2 \alpha+\rho-\alpha \rho] R-(1-\alpha)(\alpha+\rho) \frac{c}{\rho}<2 \alpha R \Leftrightarrow \frac{R}{c}<\frac{\alpha+\rho}{\rho^{2}}
$$

\section{Additional Material 2: The Suboptimal Cases Under Demand Uncertainty}

As in the basic model, again the firm can choose one of four additional cases. First, suppose the firm induces $e_{1}=1$, but $e_{2}=1$ if and only if $x_{1}=0$. That is, the sales agent should exert high effort with the second customer only if he could not sell the product to the first customer. The first-stage incentive constraint now becomes

$$
\begin{gathered}
(\alpha+\rho)\left[\beta_{1}\left(\alpha w_{11}+(1-\alpha) w_{10}\right)+\left(1-\beta_{1}\right) w_{10}\right] \\
+(1-\alpha-\rho)\left[\beta_{0}\left((\alpha+\rho) w_{01}+(1-\alpha-\rho) w_{00}-c\right)+\left(1-\beta_{0}\right) w_{00}\right]-c \\
\geq \alpha\left[\beta_{1}\left(\alpha w_{11}+(1-\alpha) w_{10}\right)+\left(1-\beta_{1}\right) w_{10}\right] \\
+(1-\alpha)\left[\beta_{0}\left((\alpha+\rho) w_{01}+(1-\alpha-\rho) w_{00}-c\right)+\left(1-\beta_{0}\right) w_{00}\right] \Leftrightarrow \\
\alpha \beta_{1} w_{11}+w_{10}\left(1-\alpha \beta_{1}\right)-\beta_{0}(\alpha+\rho) w_{01}-\left(1-(\alpha+\rho) \beta_{0}\right) w_{00} \geq \frac{1-\rho \beta_{0}}{\rho} c .
\end{gathered}
$$

The firm solves

$$
\begin{aligned}
& \min _{w_{11}, w_{10}, w_{01}, w_{00} \geq 0}(\alpha+\rho)\left[\beta_{1}\left(\alpha w_{11}+(1-\alpha) w_{10}\right)+\left(1-\beta_{1}\right) w_{10}\right] \\
& +(1-\alpha-\rho)\left[\beta_{0}\left((\alpha+\rho) w_{01}+(1-\alpha-\rho) w_{00}\right)+\left(1-\beta_{0}\right) w_{00}\right] \\
& \text { subject to } \quad w_{11}<\frac{c}{\rho}+w_{10}, \quad w_{01} \geq \frac{c}{\rho}+w_{00}, \quad \text { and }(47) .
\end{aligned}
$$

Consequently, $w_{00}=0$. Furthermore, $w_{01}$ should be as small as possible, $w_{01}=\frac{c}{\rho}$. It follows that $\beta_{1}\left(\alpha w_{11}+(1-\alpha) w_{10}\right)+\left(1-\beta_{1}\right) w_{10}=\alpha \beta_{1} w_{11}+\left(1-\alpha \beta_{1}\right) w_{10}$ should be minimal. Hence, by (47) and $w_{11}<\frac{c}{\rho}+w_{10}$, all $w_{11}$ and $w_{10}$ satisfying

$$
\alpha \beta_{1} w_{11}+\left(1-\alpha \beta_{1}\right) w_{10}=\left(1+\alpha \beta_{0}\right) \frac{c}{\rho} \text { and } w_{11}<\frac{c}{\rho}+w_{10}
$$

are optimal. The firm's profit is

$$
(\alpha+\rho)\left[\left(1+\alpha \beta_{1}+(1-\alpha-\rho) \beta_{0}\right) R-\left(1+(1-\rho) \beta_{0}\right) \frac{c}{\rho}\right]
$$

which is smaller than profit (22).

Second, consider the case where effort is high only with customer $1: e_{1}=1$ and $e_{2}=0$. The 
firm minimizes

$$
\begin{aligned}
& (\alpha+\rho)\left[\beta_{1}\left(\alpha w_{11}+(1-\alpha) w_{10}\right)+\left(1-\beta_{1}\right) w_{10}\right] \\
& +(1-\alpha-\rho)\left[\beta_{0}\left(\alpha w_{01}+(1-\alpha) w_{00}\right)+\left(1-\beta_{0}\right) w_{00}\right]
\end{aligned}
$$

subject to $w_{01}<\frac{c}{\rho}+w_{00}$ and $w_{11}<\frac{c}{\rho}+w_{10}$ as second-stage incentive constraints, and

$$
\begin{gathered}
(\alpha+\rho)\left[\beta_{1}\left(\alpha w_{11}+(1-\alpha) w_{10}\right)+\left(1-\beta_{1}\right) w_{10}\right] \\
+(1-\alpha-\rho)\left[\beta_{0}\left(\alpha w_{01}+(1-\alpha) w_{00}\right)+\left(1-\beta_{0}\right) w_{00}\right]-c \\
\geq \alpha\left[\beta_{1}\left(\alpha w_{11}+(1-\alpha) w_{10}\right)+\left(1-\beta_{1}\right) w_{10}\right] \\
+(1-\alpha)\left[\beta_{0}\left(\alpha w_{01}+(1-\alpha) w_{00}\right)+\left(1-\beta_{0}\right) w_{00}\right] \Leftrightarrow \\
\alpha \beta_{1} w_{11}+\left(1-\alpha \beta_{1}\right) w_{10}-\alpha \beta_{0} w_{01}-w_{00}\left(1-\alpha \beta_{0}\right) \geq \frac{c}{\rho}
\end{gathered}
$$

as first-stage incentive constraint. Thus, $w_{01}=w_{00}=0$, and optimal sales force compensation is described by $\beta_{1}\left(\alpha w_{11}+(1-\alpha) w_{10}\right)+\left(1-\beta_{1}\right) w_{10}=\frac{c}{\rho}$ and $w_{11}<\frac{c}{\rho}+w_{10}$. This compensation leads to profit

$$
\left((\alpha+\rho)\left(1+\alpha \beta_{1}\right)+(1-(\alpha+\rho)) \alpha \beta_{0}\right) R-(\alpha+\rho) \frac{c}{\rho},
$$

which is smaller than profit (25).

Third, the firm may be interested in implementing low effort with customer 1 and high effort with customer 2 if and only if $x_{1}=1$, that is, $e_{1}=0$ and $e_{2}=1$ iff $x_{1}=1$. The first-stage incentive constraint is

$$
\begin{gathered}
\alpha\left[\beta_{1}\left((\alpha+\rho) w_{11}+(1-\alpha-\rho) w_{10}-c\right)+\left(1-\beta_{1}\right) w_{10}\right] \\
+(1-\alpha)\left[\beta_{0}\left(\alpha w_{01}+(1-\alpha) w_{00}\right)+\left(1-\beta_{0}\right) w_{00}\right] \\
\geq(\alpha+\rho)\left[\beta_{1}\left((\alpha+\rho) w_{11}+(1-\alpha-\rho) w_{10}-c\right)+\left(1-\beta_{1}\right) w_{10}\right] \\
+(1-\alpha-\rho)\left[\beta_{0}\left(\alpha w_{01}+(1-\alpha) w_{00}\right)+\left(1-\beta_{0}\right) w_{00}\right]-c \Leftrightarrow \\
\left(1+\rho \beta_{1}\right) \frac{c}{\rho} \geq(\alpha+\rho) \beta_{1} w_{11}+\left(1-(\alpha+\rho) \beta_{1}\right) w_{10}-\alpha \beta_{0} w_{01}-\left(1-\alpha \beta_{0}\right) w_{00} .
\end{gathered}
$$

Hence, the firm's problem can be characterized as follows:

$$
\begin{gathered}
\min _{w_{11}, w_{10}, w_{01}, w_{00} \geq 0} \alpha\left[\beta_{1}\left((\alpha+\rho) w_{11}+(1-\alpha-\rho) w_{10}\right)+\left(1-\beta_{1}\right) w_{10}\right] \\
+(1-\alpha)\left[\beta_{0}\left(\alpha w_{01}+(1-\alpha) w_{00}\right)+\left(1-\beta_{0}\right) w_{00}\right] \\
\text { subject to } w_{01}<\frac{c}{\rho}+w_{00}, \quad w_{11} \geq \frac{c}{\rho}+w_{10}, \quad \text { and }(50) .
\end{gathered}
$$


It is optimal to have $w_{00}=w_{01}=w_{10}=0$ and $w_{11}=\frac{c}{\rho}$. Profit is given by

$$
\left(1+(1-\alpha) \beta_{0}+(\alpha+\rho) \beta_{1}\right) \alpha R-(\alpha+\rho) \alpha \beta_{1} \frac{c}{\rho}
$$

which is smaller than profit (17) iff $R \rho^{2}<(\alpha+\rho) c$, and smaller than profit (26) iff $R \rho^{2}>$ $(\alpha+\rho) c$.

Fourth, the firm may wish to implement low effort with customer 1 and high effort with customer 2 if and only if $x_{1}=0$, that is, $e_{1}=0$ and $e_{2}=1$ iff $x_{1}=0$. The first-stage incentive constraint reads as

$$
\begin{gathered}
\alpha\left[\beta_{1}\left(\alpha w_{11}+(1-\alpha) w_{10}\right)+\left(1-\beta_{1}\right) w_{10}\right] \\
+(1-\alpha)\left[\beta_{0}\left((\alpha+\rho) w_{01}+(1-\alpha-\rho) w_{00}-c\right)+\left(1-\beta_{0}\right) w_{00}\right] \\
\geq(\alpha+\rho)\left[\beta_{1}\left(\alpha w_{11}+(1-\alpha) w_{10}\right)+\left(1-\beta_{1}\right) w_{10}\right] \\
+(1-\alpha-\rho)\left[\beta_{0}\left((\alpha+\rho) w_{01}+(1-\alpha-\rho) w_{00}-c\right)+\left(1-\beta_{0}\right) w_{00}\right]-c \Leftrightarrow \\
\left(1-\rho \beta_{0}\right) \frac{c}{\rho} \geq \alpha \beta_{1} w_{11}+\left(1-\alpha \beta_{1}\right) w_{10}-(\alpha+\rho) \beta_{0} w_{01}-\left(1-(\alpha+\rho) \beta_{0}\right) w_{00} .
\end{gathered}
$$

The firm's problem is

$$
\begin{gathered}
\min _{w_{11}, w_{10}, w_{01}, w_{00} \geq 0} \alpha\left[\beta_{1}\left(\alpha w_{11}+(1-\alpha) w_{10}\right)+\left(1-\beta_{1}\right) w_{10}\right] \\
+(1-\alpha)\left[\beta_{0}\left((\alpha+\rho) w_{01}+(1-\alpha-\rho) w_{00}\right)+\left(1-\beta_{0}\right) w_{00}\right] \\
\text { subject to } w_{01} \geq \frac{c}{\rho}+w_{00}, \quad w_{11}<\frac{c}{\rho}+w_{10}, \quad \text { and }(51) .
\end{gathered}
$$

It is thus optimal to have $w_{00}=w_{10}=w_{11}=0$ and $w_{01}=\frac{c}{\rho}$, leading to profit

$$
\left(\alpha+((1-(\alpha+\rho)) \alpha+\rho) \beta_{0}+\alpha^{2} \beta_{1}\right) R-\beta_{0}(1-\alpha)(\alpha+\rho) \frac{c}{\rho},
$$

which is smaller than profit (17) iff $R \rho^{2}<(\alpha+\rho) c$, and smaller than profit (26) iff $R \rho^{2}>$ $(\alpha+\rho) c$.

\section{Additional Material 3: The Suboptimal Cases Under Preference Uncertainty}

First, suppose the firm wants to induce $e_{1}=1$, but $e_{2}=1$ if and only if $x_{1}=0$. The first-stage incentive constraint becomes

$$
E[\rho \alpha]\left(w_{11}-w_{10}\right)+E[\rho]\left(w_{10}-w_{00}\right)-E[\rho(\alpha+\rho)]\left(w_{01}-w_{00}\right) \geq(1-E[\rho]) c
$$


and the firm minimizes

$$
\begin{gathered}
E\left[(\alpha+\rho) \alpha w_{11}+(\alpha+\rho)(1-\alpha) w_{10}+(1-\alpha-\rho)(\alpha+\rho) w_{01}+(1-\alpha-\rho)^{2} w_{00}\right] \\
\text { subject to } \quad w_{11}<\frac{c}{E[\rho]}+w_{10}, \quad w_{01} \geq \frac{c}{E[\rho]}+w_{00}, \quad \text { and }(52) .
\end{gathered}
$$

Hence, $w_{00}=0$ and $w_{01}=c / E[\rho]$. The firm minimizes costs $C$ characterized by the iso-cost curves

$$
\frac{C}{B_{2}}-\frac{A_{2}}{B_{2}} \frac{c}{E[\rho]}-\frac{B_{1}}{B_{2}} w_{11}=w_{10}
$$

subject to the constraints

$$
\begin{aligned}
w_{11}-\frac{c}{E[\rho]} & <w_{10} \\
\frac{(1-E[\rho])+\frac{A_{3}}{E[\rho]}}{E[\rho]-E[\rho \alpha]} c-\frac{E[\rho \alpha]}{E[\rho]-E[\rho \alpha]} w_{11} & \leq w_{10}
\end{aligned}
$$

with $B_{1}:=E[(\alpha+\rho) \alpha], B_{2}:=E[(\alpha+\rho)(1-\alpha)]$, and $A_{2}$ and $A_{3}$ as defined above. The LHS of (54) intersects with the horizontal axis at $w_{11}=c / E[\rho]$. The LHS of (55) intersects with the horizontal axis at ${ }^{23}$

$$
w_{11}=\frac{(1-E[\rho])+\frac{A_{3}}{E[\rho]}}{E[\rho \alpha]} c>\frac{c}{E[\rho]} .
$$

The LHSs of both constraints (54) and (55) intersect at

$$
\hat{w}_{11}:=\frac{(1-p) p\left(\rho_{1}-\rho_{2}\right)^{2}+2 E[\rho]}{E[\rho]^{2}} c \text { and } \hat{w}_{10}:=\frac{(1-p) p\left(\rho_{1}-\rho_{2}\right)^{2}+E[\rho]}{E[\rho]^{2}} c .
$$

Since $E[\rho \alpha] /(E[\rho]-E[\rho \alpha])=\alpha /(1-\alpha)=B_{1} / B_{2}$, the slopes of the iso-cost curves and the LHS of (55) are identical. Therefore, each wage combination that lies on the LHS of (55) between the points $\left(w_{11}, w_{10}\right)=\left(0,\left[(1-E[\rho])+\frac{A_{3}}{E[\rho]}\right] c /[E[\rho]-E[\rho \alpha]]\right)$ and $\left(w_{11}, w_{10}\right)=\left(\hat{w}_{11}, \hat{w}_{10}\right)$ describes an optimal solution, leading to profits

$$
\begin{aligned}
\pi_{f i r s t} & =E[\alpha+\rho] R+\{E[(\alpha+\rho) \alpha]+E[(1-\alpha-\rho)(\alpha+\rho)]\} R \\
& -E[(\alpha+\rho) \alpha] \frac{(1-p) p\left(\rho_{1}-\rho_{2}\right)^{2}+2 E[\rho]}{E[\rho]^{2}} c \\
& -E[(\alpha+\rho)(1-\alpha)] \frac{(1-p) p\left(\rho_{1}-\rho_{2}\right)^{2}+E[\rho]}{E[\rho]^{2}} c \\
& -\frac{E[(1-\alpha-\rho)(\alpha+\rho)]}{E[\rho]} c .
\end{aligned}
$$

${ }^{23}$ Note that $\frac{(1-E[\rho])+\frac{A_{3}}{E[\rho]}}{E[\rho \alpha]} c>\frac{c}{E[\rho]} \Leftrightarrow(1-E[\rho]) E[\rho]+E[\rho(\alpha+\rho)]>E[\rho \alpha]$ is true since $E[\rho(\alpha+\rho)]>E[\rho \alpha]$. 
We can show that $\pi_{(I I)}>\pi_{\text {first }}$ is always satisfied:

$$
\begin{gathered}
\pi_{(I I)}>\pi_{\text {first }} \\
\Leftrightarrow E\left[\rho^{2}\right] R+E[\alpha \rho] R-\frac{E[\alpha+\rho]}{E[\rho]} c-\frac{E\left[(\alpha+\rho)^{2}\right]}{E[\rho(\alpha+\rho)]} c \\
>-E[(\alpha+\rho) \alpha] \frac{(1-p) p\left(\rho_{1}-\rho_{2}\right)^{2}+2 E[\rho]}{E[\rho]^{2}} c \\
-E[(\alpha+\rho)(1-\alpha)] \frac{(1-p) p\left(\rho_{1}-\rho_{2}\right)^{2}+E[\rho]}{E[\rho]^{2}} c \\
-\frac{E[(1-\alpha-\rho)(\alpha+\rho)]}{E[\rho]} c .
\end{gathered}
$$

Recall that exerting high effort is efficient, i.e., $E[\rho] R>c$. Inserting $R=c / E[\rho]$ into the inequality yields

$$
\frac{p\left(\rho_{1}-\rho_{2}\right)^{2}(1-p) \Omega c}{\left(p \rho_{1}+(1-p) \rho_{2}\right)^{2}\left(p \rho_{1}^{2}+(1-p) \rho_{2}^{2}+\alpha\left(p \rho_{1}+(1-p) \rho_{2}\right)\right)}>0
$$

with $\Omega:=\alpha\left(p \rho_{1}\left(1+(1+p) \rho_{1}\right)+\left(p^{2}+2-3 p\right) \rho_{2}^{2}\right)+(1-p)^{2} \rho_{2}^{3}+p^{2} \rho_{1}^{3}+\alpha^{2}\left(p \rho_{1}+(1-p) \rho_{2}\right)+$ $\left(\left(\rho_{2}+\rho_{1}+2 \alpha\right) p \rho_{1} \rho_{2}+\alpha \rho_{2}\right)(1-p)$, which is true.

Second, consider the case where effort is high only with one customer: $e_{i}=1$ and $e_{j}=0$ $(i, j=1,2, i \neq j)$. Let, w.l.o.g., $i=1$. The firm minimizes

$$
E\left[(\alpha+\rho) \alpha w_{11}+(\alpha+\rho)(1-\alpha) w_{10}+(1-\alpha-\rho) \alpha w_{01}+(1-\alpha-\rho)(1-\alpha) w_{00}\right]
$$

subject to $w_{01}<\frac{c}{E[\rho]}+w_{00}$ and $w_{11}<\frac{c}{E[\rho]}+w_{10}$ as second-stage incentive constraints, and

$$
\alpha w_{11}+(1-\alpha) w_{10}-\alpha w_{01}-(1-\alpha) w_{00} \geq \frac{c}{E[\rho]}
$$

as first-stage incentive constraint. Thus, $w_{01}=w_{00}=0$ is optimal. The firm minimizes costs $C$ described by the iso-cost curves

$$
\begin{gathered}
\frac{C}{E[(\alpha+\rho)(1-\alpha)]}-\frac{\alpha}{1-\alpha} w_{11}=w_{10} \\
\text { subject to } \frac{c}{(1-\alpha) E[\rho]}-\frac{\alpha}{1-\alpha} w_{11} \leq w_{10} \text { and } w_{11}-\frac{c}{E[\rho]}<w_{10} .
\end{gathered}
$$

Since the slopes of the iso-cost curves and the LHS of the first constraint are identical, we obtain a similar solution as in the first additional case above. For example, $w_{11}=0$ and 
$w_{10}=c /[(1-\alpha) E[\rho]]$ is optimal. Each solution leads to expected profits

$$
\pi_{\text {second }}=E[\alpha+\rho] R+\alpha R-\frac{E[(\alpha+\rho)(1-\alpha)] c}{(1-\alpha) E[\rho]} .
$$

We can show that $\pi_{(I I I)}>\pi_{\text {second }}$ :

$$
\begin{gathered}
\pi_{(I I I)}>\pi_{\text {second }} \\
\Leftrightarrow\left(p \rho_{1}^{2}+(1-p) \rho_{2}^{2}+p \alpha \rho_{1}+(1-p) \alpha \rho_{2}\right) R-\frac{E\left[(\alpha+\rho)^{2}\right](1+E[\rho]) c}{E[\rho(\alpha+\rho)]} \\
>-\frac{E[(\alpha+\rho)(1-\alpha)] c}{(1-\alpha) E[\rho]} .
\end{gathered}
$$

Inserting $R=c / E[\rho]$ and rearranging gives

$$
\frac{p\left(\rho_{1}-\rho_{2}\right)^{2}(1-p)\left(\alpha+p \rho_{1}^{2}+(1-p) \rho_{2}^{2}+2 \alpha\left(p \rho_{1}+(1-p) \rho_{2}\right)\right) c}{\left(p \rho_{1}+(1-p) \rho_{2}\right)\left(p \rho_{1}^{2}+(1-p) \rho_{2}^{2}+p \alpha \rho_{1}+(1-p) \alpha \rho_{2}\right)}>0,
$$

which is true.

Third, the firm may be interested in implementing low effort with customer 1 and high effort with customer 2 if and only if $x_{1}=1$, that is, $e_{1}=0$ and $e_{2}=1$ iff $x_{1}=1$. The first-stage incentive constraint is

$$
(1+E[\rho]) c \geq E\left(\rho\left[(\alpha+\rho) w_{11}+(1-\alpha-\rho) w_{10}\right]-\rho\left[\alpha w_{01}+(1-\alpha) w_{00}\right]\right) .
$$

Hence, the firm's problem can be characterized as follows:

$$
\begin{aligned}
& \min _{w_{11}, w_{10}, w_{01}, w_{00} \geq 0} E\left[\alpha(\alpha+\rho) w_{11}+\alpha(1-\alpha-\rho) w_{10}+(1-\alpha) \alpha w_{01}+(1-\alpha)^{2} w_{00}\right] \\
& \text { subject to } w_{01}<\frac{c}{E[\rho]}+w_{00}, \quad w_{11} \geq \frac{c}{E[\rho]}+w_{10}, \quad \text { and }(56) .
\end{aligned}
$$

It is optimal to have $w_{10}=0$ and $w_{11}=\frac{c}{E[\rho]}$. The firm minimizes costs $C$ described by the iso-cost curves

$$
\begin{gathered}
\frac{C}{(1-\alpha)^{2}}-\frac{E[\alpha(\alpha+\rho)]}{(1-\alpha)^{2} E[\rho]} c-\frac{\alpha}{1-\alpha} w_{01}=w_{00} \quad \text { subject to } \\
\left(\frac{E[\rho(\alpha+\rho)]}{E[\rho]}-(1+E[\rho])\right) \frac{c}{E[\rho(1-\alpha)]}-\frac{\alpha}{1-\alpha} w_{01} \leq w_{00} \quad \text { and } \quad w_{01}-\frac{c}{E[\rho]}<w_{00} .
\end{gathered}
$$

Note that, again, the iso-cost curves and the LHS of the first-stage incentive constraint have identical slopes. However, now we have two possible solutions: (i) If the intercept of the LHS 
of the first-stage incentive constraint is negative, i.e., if

$$
\left(\frac{E[\rho(\alpha+\rho)]}{E[\rho]}-(1+E[\rho])\right) \frac{c}{E[\rho(1-\alpha)]}<0 \Leftrightarrow \frac{(1-p) p\left(\rho_{1}-\rho_{2}\right)^{2}}{p \rho_{1}+(1-p) \rho_{2}}<1-\alpha,
$$

then $w_{01}=w_{00}=0$ is optimal and expected profits amount to

$$
\pi_{t h i r d,(i)}=\alpha R+\{E[\alpha(\alpha+\rho)]+(1-\alpha) \alpha\} R-E[\alpha(\alpha+\rho)] \frac{c}{E[\rho]} .
$$

(ii) If (58) is not satisfied, then independent of the relation of the zeros of the two incentive constraints in (57), the pair

$$
w_{01}=0 \quad \text { and } \quad w_{00}=\left(\frac{E[\rho(\alpha+\rho)]}{E[\rho]}-(1+E[\rho])\right) \frac{c}{E[\rho(1-\alpha)]}
$$

describes an optimal solution, leading to expected profits

$$
\begin{aligned}
\pi_{t h i r d,(i i)} & =\alpha R+\{E[\alpha(\alpha+\rho)]+(1-\alpha) \alpha\} R \\
& -E[\alpha(\alpha+\rho)] \frac{c}{E[\rho]}-\left(\frac{E[\rho(\alpha+\rho)]}{E[\rho]}-(1+E[\rho])\right) \frac{(1-\alpha)^{2} c}{E[\rho(1-\alpha)]}
\end{aligned}
$$

Since $\pi_{t h i r d,(i)}>\pi_{t h i r d,(i i)}$, the third additional case cannot be optimal if one of the profits of the other cases exceeds $\pi_{\text {third,(i) }}$. Inducing no incentives at all is better for the firm than the third additional case, iff

$$
\begin{aligned}
\pi_{\text {third, }(i)} & =\alpha R+\{E[\alpha(\alpha+\rho)]+(1-\alpha) \alpha\} R-E[\alpha(\alpha+\rho)] \frac{c}{E[\rho]}<2 \alpha R \\
& \Leftrightarrow E[\rho] R<E[\alpha+\rho] \frac{c}{E[\rho]} .
\end{aligned}
$$

The firm will prefer the second additional case to the third additional case, iff

$$
\pi_{\text {second }}>\pi_{\text {third },(i)} \Leftrightarrow E[\rho] R>E[\alpha+\rho] \frac{c}{E[\rho]} .
$$

Thus, the firm does not prefer the third additional case.

Fourth, the firm may wish to implement low effort with customer 1 and high effort with customer 2 if and only if $x_{1}=0$, that is, $e_{1}=0$ and $e_{2}=1$ iff $x_{1}=0$. The first-stage incentive constraint reads as

$$
(1-E[\rho]) c \geq E\left[\rho\left(\alpha w_{11}+(1-\alpha) w_{10}-(\alpha+\rho) w_{01}-(1-\alpha-\rho) w_{00}\right)\right] .
$$


The firm solves

$$
\begin{aligned}
\min _{w_{11}, w_{10}, w_{01}, w_{00} \geq 0} E\left[\alpha^{2} w_{11}+\alpha(1-\alpha) w_{10}+(1-\alpha)(\alpha+\rho) w_{01}+(1-\alpha)(1-\alpha-\rho) w_{00}\right] \\
\text { subject to } \quad w_{01} \geq \frac{c}{E[\rho]}+w_{00}, \quad w_{11}<\frac{c}{E[\rho]}+w_{10}, \quad \text { and }(59) .
\end{aligned}
$$

It is thus optimal to have $w_{10}=w_{11}=w_{00}=0$ and $w_{01}=c / E[\rho]$. Expected profit is

$$
\pi_{\text {fourth }}=\alpha R+\alpha^{2} R+(1-\alpha) E[\alpha+\rho] R-(1-\alpha) E[\alpha+\rho] \frac{c}{E[\rho]} .
$$

Since $\pi_{\text {fourth }}<2 \alpha R \Leftrightarrow E[\rho] R<E[\alpha+\rho] c / E[\rho]$ and $\pi_{\text {fourth }}<\pi_{\text {second }} \Leftrightarrow E[\alpha+\rho] c / E[\rho]<$ $E[\rho] R$, the firm does not prefer the fourth additional case. 